\title{
THE TRANSITION EXPERIENCE OF FACULTY MEMBERS INTO ACADEMIC LEADERSHIP
}

A Dissertation
presented to
the Faculty of the Graduate School
at the University of Missouri-Columbia
In Partial Fulfillment
of the Requirements for the Degree
Doctor of Philosophy
Dr. Bradley Curs, Dissertation Supervisor
MATIN QAZI
by

December 2020 
The undersigned, appointed by the dean of the Graduate School, have examined the dissertation entitled

THE TRANSITION EXPERIENCE OF FACULTY MEMBERS INTO ACADEMIC

LEADERSHIP

presented by Matin Qazi,

a candidate for the degree of Doctor of Philosophy of Educational leadership and policy analysis and hereby certify that, in their opinion, it is worthy of acceptance.

Professor Bradley Curs

Professor Jeni Hart

Professor Pilar Mendoza

Professor Tony Castro 


\section{DEDICATION}

To my family. 


\section{ACKNOWLEDGEMENT}

First and foremost, I am grateful to my advisor Dr. Bradley Curs. You admitted me to the program and advised me throughout the years. Your mentorship has helped me to grow both academically and professionally. Thank you for believing in me, teaching me and empowering me. This PhD journey would not be the same without you.

Next, I would like to thank my committee members. Dr. Jeni Hart, I have learned from every interaction with you. Your knowledge and wisdom are impressive. Thank you for all your feedback to enrich and improve my work. Dr. Pilar Medoza, I owe much of my growth over the years to your encouragement. Thank you for taking interest in my progress as a student and researcher. Dr. Tony Castro deserves the utmost appreciation for stepping in to sit on my committee at the last minute, and for trusting my quality of work and scholarship.

I am grateful to the seven chairs who participated in this study despite their very busy work schedule. You vulnerably shared your stories and allowed me to study your transition experiences. Last but not least, I cannot overstate how much my friends and family have done to achieve this goal. I am grateful for all their support, encouragement, and love during my doctoral career. 


\section{TABLE OF CONTENTS}

Acknowledgments ............................................................... ii

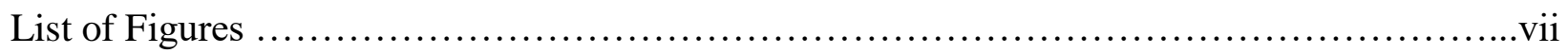

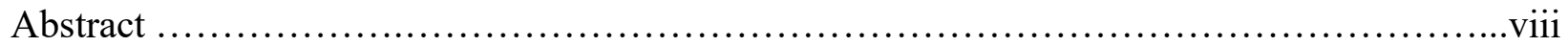

CHAPTER 1: OVERVIEW ....................................................................................... 1

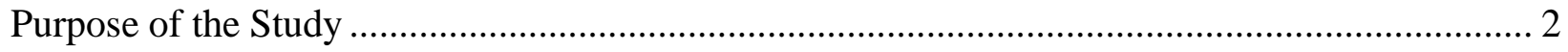

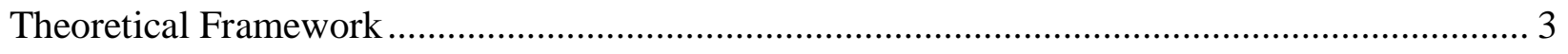

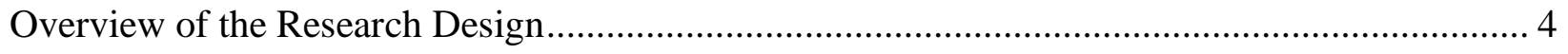

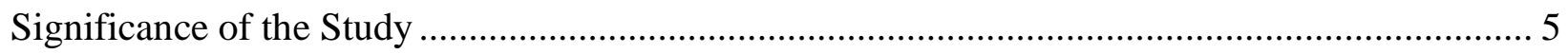

CHAPTER 2: REVIEW OF THE LITERATURE …...................................................... 7

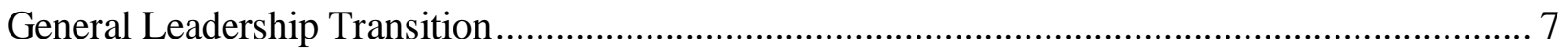

Transition of an Expert into Leadership .................................................................. 7

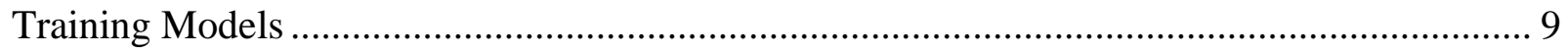

Need Assessment................................................................................................ 12

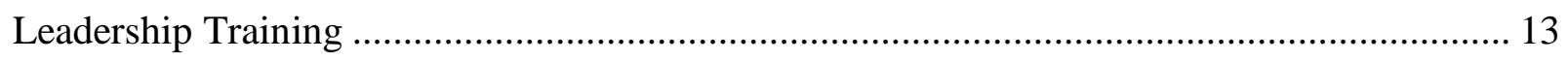

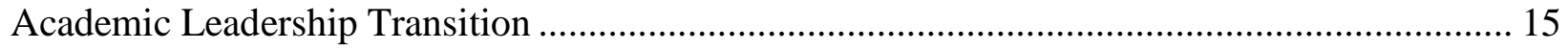

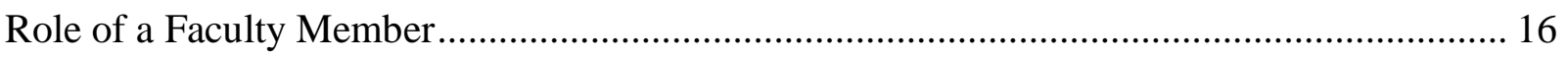

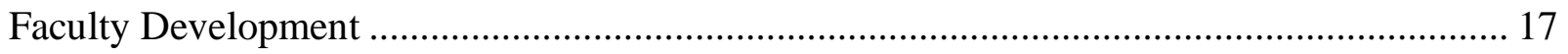

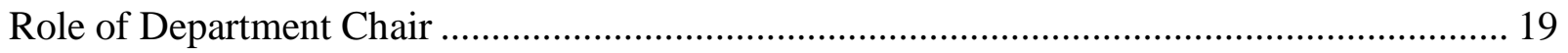

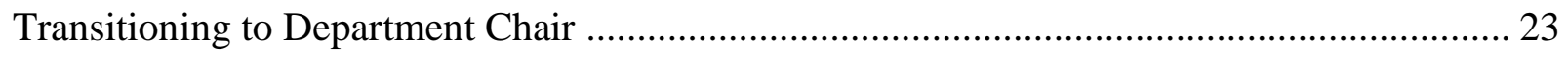

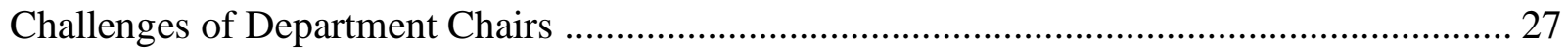

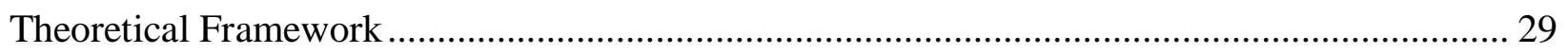




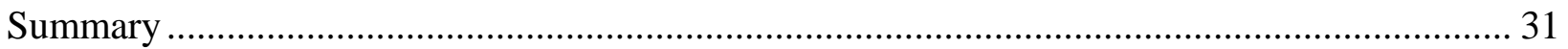

CHAPTER 3: RESEARCH DESIGN AND METHODOLOGY ........................................... 33

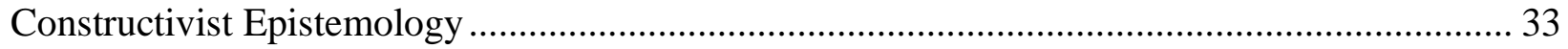

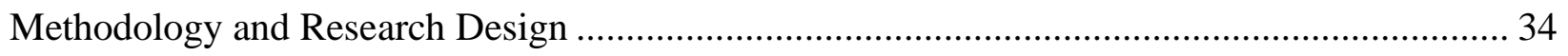

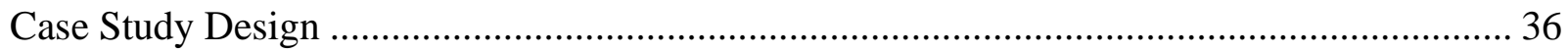

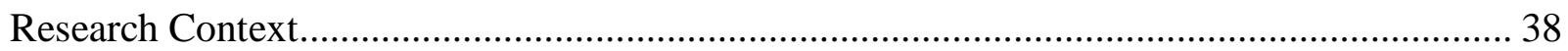

Participants and Selection Procedures............................................................................... 39

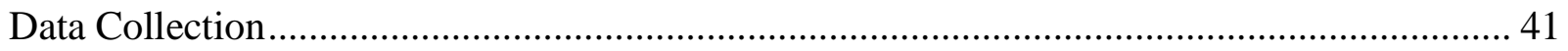

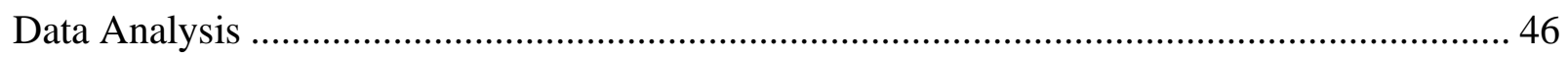

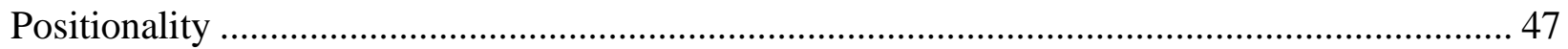

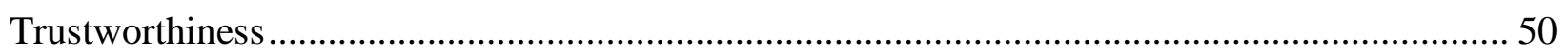

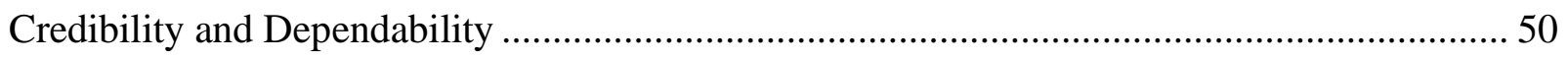

Confirmability and Transferability ....................................................................... 51

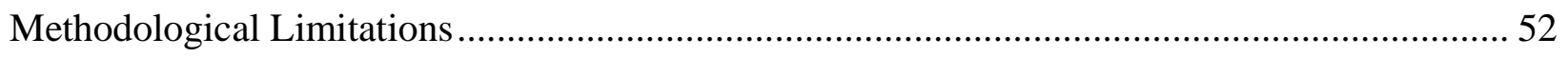

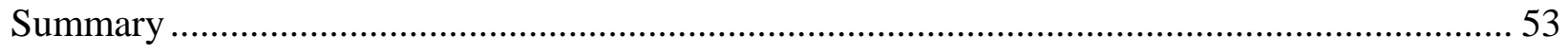

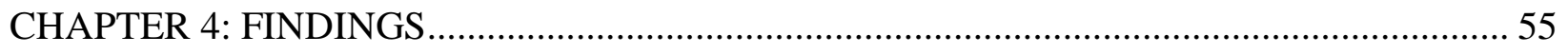

Theme I: Transition is a Multifaceted Departure ............................................................ 57

From Faculty to Chair: Transformation of Career Motivations ......................................... 57

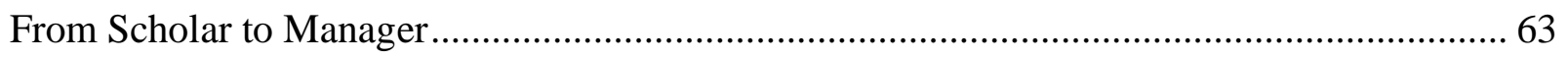

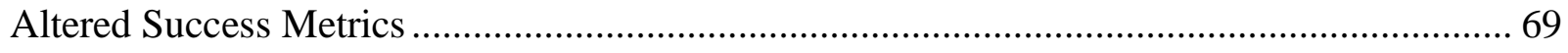

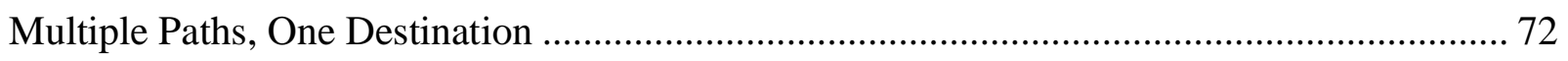




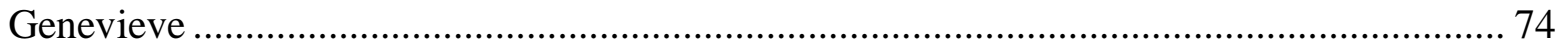

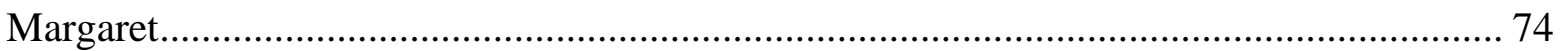

Theodore

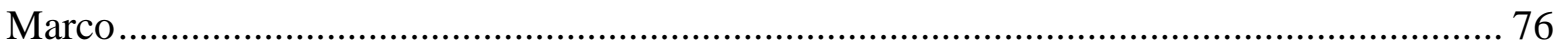

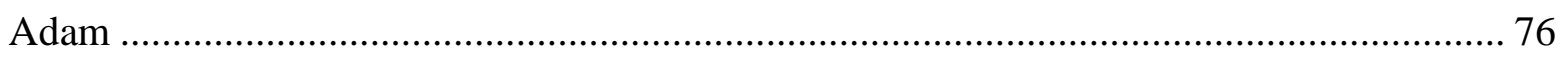

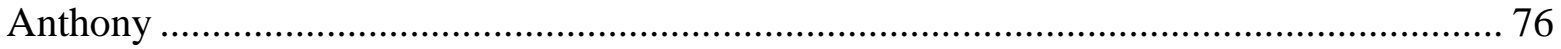

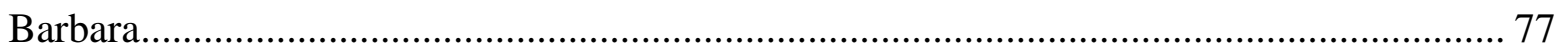

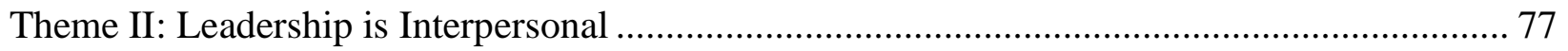

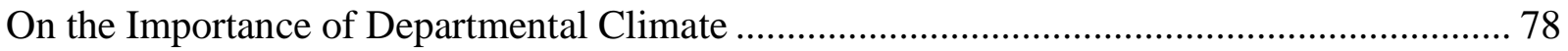

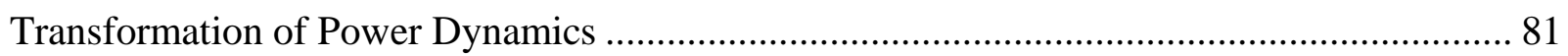

On the Importance of Interpersonal Skills ............................................................................ 84

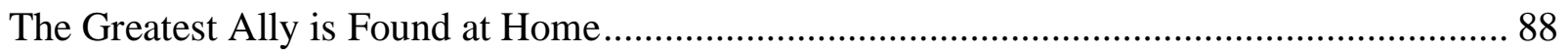

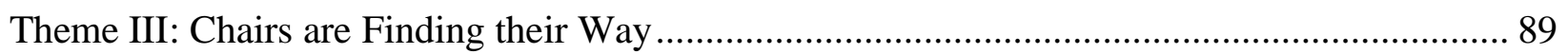

Service Provided an Initial Window into Academic Leadership ............................................... 90

The Predecessor's Role in Shaping the Transition.................................................................... 92

Trial by Fire: Lack of Preparation .................................................................................... 95

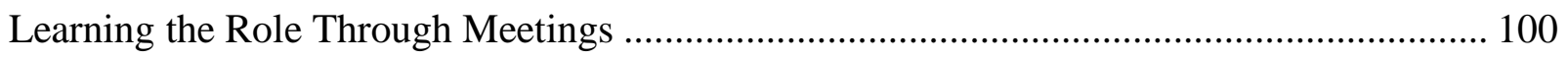

Solving Problems through Alliances and Consultations ...................................................... 102

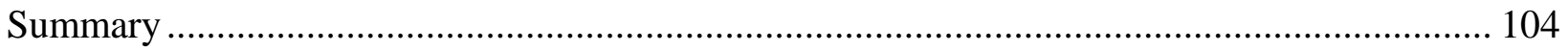

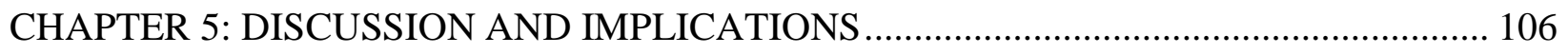

Transition Experience to Leadership ................................................................................... 107

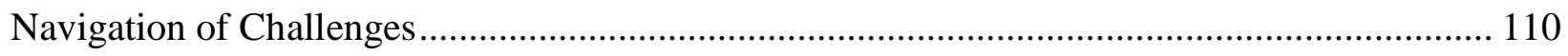




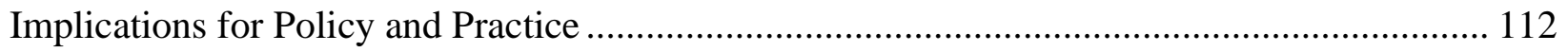

Implications for Further Research ............................................................................ 116

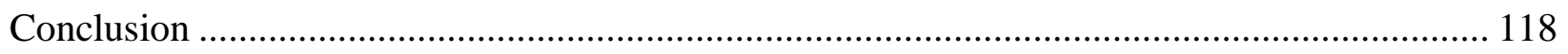

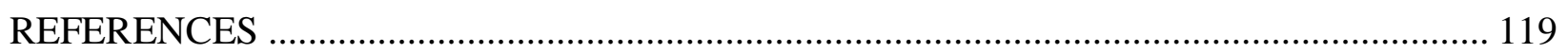

APPENDIX I: INVITATION EMAIL .................................................................... 126

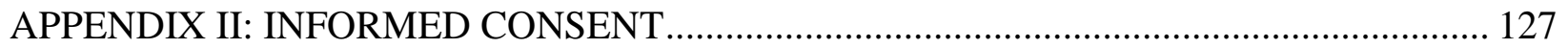

APPENDIX III: INTERVIEW PROTOCOL: SESSION I .............................................. 129

APPENDIX IV: INTERVIEW PROTOCO- SESSION II ............................................. 130

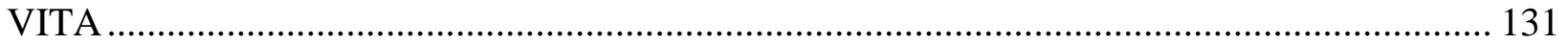




\section{LIST OF FIGURES}

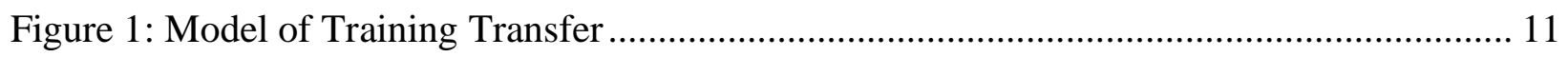




\begin{abstract}
The purpose of this case study was to understand the lived experiences of faculty members as they transitioned into the academic leadership role of chair. The case study design was used to provide an intensive bounded analysis of the real-life transition experiences of new chairs. Seven new chairs provided data through two interviews each and documents including their resumés, calendars, and appointment letters. By bounding the case study to the University of Missouri, the data provided a rich dataset which captured the collective experience of transitioning into academic leadership, the complexities of their transitions, and how they navigate the transitional challenges. Three primary themes emerged from the data: Theme I: Transition is a Multifaceted Departure, Theme II: Leadership is Interpersonal, and Theme III: Chairs are Finding their Way. I delineated the collective experience of chairs at the University of Missouri to leadership. I reflected their strategies to navigate this path and discussed the resources that are provided to support them along the way. This increased understanding of the transition process and its associated challenges will improve the policy and practice in the appointment of department chairs, and it will inform perspective chairs of the obstacles that other chairs have experienced. The major policy implications of this study are that earlier appointment of chairs provides more time to prepare for the role and allows greater time to design a succession plan with collaborating predecessors. In addition, the leadership should provide needbased support system on the required competency areas for incoming chairs.
\end{abstract}




\section{CHAPTER 1: OVERVIEW}

Department chairs hold influential roles of authority and decision making in higher education in the United States. With the chair role comes substantial responsibility, as they have the power to shape the policies and practices within their departments and colleges (Berdrow, 2010). This singular role in higher education collaborates with and influences many stakeholders including faculty, staff, students and applicants, alumni, academic leaders and administrators, and various other local and non-local community members. Additionally, chairs affect recruitment, retention, excellence, and placement of their departments' students (Wolverton, Ackerman, \& Holt, 2005).

Despite the strategic importance of this particular role in the higher education system, many of the faculty members who embark on this new career are unprepared and poorly trained for the role (Glick, 2006). As a novice department leader, this unique experience of transitioning from professorship to a leadership position demands the mastery of a distinct skillset (Foster, 2006). As Hecht (2001) asserted, chairs find themselves "operating in a murky landscape of either ill-defined or conflicting expectations" (p. 7).

Gmelch and Parkay (1999) called the transition from a faculty to chair a metamorphosis and they created a taxonomy of characteristics to describe how chairs adjust during their transition period. They found that college professors spend most of their time working alone with flexible working conditions, while a department chair to require to constantly work with others, often with a lesser degree of autonomy and flexibility. Departmental leaders are also directly accountable to their supervisors, faculty, and students. Chairs must learn the art of persuasion and precision, and act as a custodians and managers of the resources of the university. This 
taxonomy illustrates the transformation of a faculty members life and how new responsibilities are thrust upon them.

Chairs may be unaware of the toll this position will take on their academic career and personal lives (Gmelch, 2004). In an autoethnographic study, Palm (2006) reflected back on her transition from faculty to administration. She described her transition to administration as moving to "the dark side." Before officially accepting the position, Palm perceived her new job as a partial sabbatical from teaching. She thought the transition to the department chair seemed deceptively easy initially, but later discovered it to be nothing like what she originally thought. Eventually, Palm came to the conclusion that academic leadership is a position for a faculty member who is willing to sacrifice their own academic career to advance the goals of the department and college.

\section{Purpose of the Study}

In this study, I explore transition experience of professors into leadership and investigate how chairs learn the nuts and bolts of their new position at the University of Missouri. The purpose of this research is to unveil how faculty members experience their transition into departmental leadership. In this case study, I will attempt to focus the spotlight on chairs and their academic and professional settings at the University of Missouri, and I will reveal their sensemaking of their experiences. While I probe the transition process, my focus will remain on the competency challenges and chairs approaches to overcome them.

In this research, I address the following two research questions:

1. How do new department chairs experience the transition from a faculty position to a leadership role? 
2. How do new department chairs navigate the challenges of the transitioning to leadership?

\section{Theoretical Framework}

The conceptual framework of this study is built on the transition theory of Schlossberg (1981, 1995, 2011). Schlossberg $(1995,2011)$ suggested that there are four systems that affect the coping mechanisms during the transition process: situation, self, supports, and strategies. The individuals' situations at the time of transition affects the transition experience. Moreover, individual's inner strength for coping with transition varies and is an important factor that must be considered. Another major difference is the presence and type of support. Finally, the strategies utilized to cope with the transition are important. For instance, some may talk to a professional consultant while others prefer to brainstorm or join a club to cope with the change.

Schlossberg (1995) believes that no two transitions are exactly alike; however, transitions can be categorized into three groups: anticipated transition, unanticipated transition, and nonevent transition. Anticipated transition, as the name implies, happens when the career shift is expected in advance. In unanticipated transition, the transition happens unexpectedly and usually in a disruptive manner. Non-event transition occurs when the expected event fails to happen. For example, a person who expects to be promoted does not get the promotion. All of these transitions have some elements in common. First, the transition changes an individual's routines, assumptions, and roles. Second, although the transitions may be abrupt, the individual and personal changes take time and their reaction to the change will also evolve as time passes. Finally, people differ from one another in how they manage the transition and are able to cope with it. This transition model provides the structure to study and analyze transitions. 


\section{Overview of the Research Design}

This research is inspired by the philosophical paradigm of constructivism and delves into the sense making process of the faculty during their transition into department chairs. Specifically, I use the case study method from a constructivist epistemology where the truth is relative and dependent on one's perspective. I attempt to acknowledge subjective human sense making, without utterly rejecting the objectivity of the experience. Baxter and Jack (2008) assert that one advantage of the constructivist approach to case studies is the close interaction between researcher and participants, enabling them to disclose their experiences and share their stories. In this process, I chose to observe the reality from the participant point of view to better grasp the understanding of the phenomenon and the lived experience. Therefore, this epistemological approach meshes perfectly with the primary research interest to understand the meaning and reconstruct the knowledge and reality of the people who are undergoing a certain experience.

I utilize the constructivism paradigm in conjunction with the case study design to explore the complexities of the transitions to a department chair. Constructivism takes into consideration unique experiences, and thanks to case study design, allows me to study the role of their context in shaping the subjective reality of new chairs in the transition phase. I will provide a rich description of the processes and challenges to explore the commonalities and differences of department chair transition. I chose an embedded single-case design for this research.

I approach this study from a qualitative perspective to gain an in-depth understanding of the experience of transition, its complexities, and the navigation of its challenges. This case study is bounded analyses of the real-life transition experience of chairs at University of Missouri. The case study design enabled me to understand the real-life transition experience of the faculty members at this institution. I collected data from seven chairs throughout two 
interviews with each participant and analyzed their resumes, calendars, and appointment letters. I chose multiple units of analysis to incorporate multiple angles to investigate the issues in order to reach greater understanding of department chairs transition experience.

\section{Significance of the Study}

Typically, professors and scholars temporarily accept the responsibilities of a department chair despite not having leadership training, administrative experience, or a clear understanding

of the complexity of the role (Gmelch, 1991). Transitioning into a leadership in academia, sparks new relationship with multiple constituencies and alters old ones for the faculty members. Smith and Stewart (1999) found that $41 \%$ did not stay longer than 2 years in the position and $63 \%$ of chairs stated that they would not recommend a chair position to others. Carroll and Wolverton (2004) suggested that "dealing with people, coping with the bureaucracy of the institution, and the negative impact on research productivity" (p. 7) are the three major stress-generating categories for a department chair. Along a similar vein, Gmelch (1991) found that managerial stressors such as complying with rules and resolving collegial issues led to $20 \%$ more emotional stress for the department chairs compared to faculty members. Just as department chairs begin learning the ropes, they usually move back to faculty positions (Thomas \& Schuh, 2004).

This study is an attempt to reconstruct the department chairs transition experience. Previous studies in this domain, such as Foster (2006) and Bowman (2002) and Thomas and Schuh (2004), offered recommendations on how to be an effective department chair based on their own experience as a chair; however, the authors did not empirically research the process. The lessons learned from the experience of department chairs in transition can inform the other junior chairs about the challenges of transitions and how they are dealing with it. 
While work satisfaction, role challenges, deficiencies, and recommended best practices for department chairs have been previously addressed by scholars (Gillet-Karam, 1999; Hubbell, \& Homer, 1997; Seagren,1993; Thomas \& Schuh 2004), the empirical research on the transition of the department chair is inadequate and limited to some autoethnographies. To my knowledge, no study has focused on the empirical reconstruction of the transition experience of first-time chairs. Competency challenges, skill deficiency and newly incumbent chair strategies to overcome them are all still nebulous.

Increased understanding of the transition process and its associated challenges will improve the policy and practice in the appointment of department chairs, and it will inform perspective chairs of the obstacles that other chairs have experienced. This knowledge of potential challenges will help perspective chairs or a newly incumbent chairs to better prepare and has the potential to facilitate the transition process. Due to the complexity of the department chair role, and lack of prior study in this regard, I will narrow this paper by proposing a research design to address the issue empirically. The outcome of the research suggests reforms needed in the area of competency development that will affect existing policies and practices. I believe recommending professional development agendas, allocating resources, or timelines for phasing the implementation would be premature at this stage. 


\section{CHAPTER 2: REVIEW OF THE LITERATURE}

In this chapter, I will review the pertinent literature of the transition to academic leadership. I organize this literature review into three research areas: general leadership transition, academic leadership transition, and the theoretical framework. These three domains will provide the background and framework supporting this study. With respect to the research questions, I put greater focus on competency challenges and training needs when reviewing leadership and department chair transition. In the last section of this chapter, I introduce the Schlossberg's $(1981,1995,2011)$ transition theory as the conceptual framework of this study.

\section{General Leadership Transition}

In this section, I review the literature on general leadership transition issues. Exploring the transition of experts into leadership positions will elucidate the pivotal issues of leadership transition. I discuss the experience of transition, leadership challenges, needs, and leadership development programs. This holistic understanding informs the more focused experience of academic leadership transitions.

\section{Transition of an Expert into Leadership}

The transition of a field expert to manager is not limited to higher education settings; this phenomenon is quite prevalent outside of academia as well. For example, at some point in their careers, nearly half of all engineers move to some form of a managerial position (Biddle \& Roberts, 1994). This track switching is commonly perceived as career advancement, and it is usually associated with higher earning. Howard (2003) stated that transformation of engineers into managers is one of the most formidable challenges in their organizations, but at the same time, is vital for their survival and growth. The skills required to be an effective engineer are quite different from those of an effective engineering manager. Engineering requires a 
considerable amount of technical knowledge and training. With many engineers believing the transition to management a waste of their potential and training, they are inherently more inclined toward pursuing technical activities rather than managerial and leadership roles (Johnson \& Sargeant, 1998). Many engineers find it difficult to make the transition to leadership and managerial positions (Badaway, 1995; Howard, 2003).

There is a trend for skilled, high-earning technical workers to flourish in management, with greater success in managerial positions being related to previous success in technical positions. Biddle and Roberts (1994) conducted a regression analysis on the National Science Foundation's (NSF) longitudinal survey of natural scientists and engineers and found that technical and managerial abilities positively correlate with each other. While the correlation is not perfect, it indicated that performance in a technical track predicts the potential in a management track. Biddle and Roberts modeled track switching as a process of self-selection by private sector scientists and engineers and concluded that track switching may be part of the process of human capital investment.

Howard (2003) studied the challenges of engineers moving from the role of an engineer to a manager through qualitative interviews. He found that in the transition process, engineering managers become overwhelmed with balancing sundry responsibilities as they were not accustomed to doing so in their previous career track. Beyond the overwhelming task load, managing interaction dynamics and personal relationships at work were the managers' primary concern. Another common theme that he described among new engineering managers was their challenges in delegating activities to technical workers and other subordinates. Mahlangu (2015) found that the 
most important competency deficiencies of technicians in a supervisory role were interpersonal relations, leadership behaviors, organization ability, and budgeting.

One of the reasons for the lack of expertise and transition challenges from engineering to managerial positions was the scope of the training programs. Johnson and Sargeant (1998) stated that university engineering programs are strictly narrowed to technical disciplines and the skills needed to perform their functional roles in the profession. Another challenging aspect of the transition are the idiosyncrasies of the junior managers and their working environment.

Nicholson (1984) suggested that personal adjustments to their role transition vary from person to person based on their self-concept, values, skills, and lifestyle. For instance, proactive individuals may engage actively in the process of role development to change their role requirements to better fit themselves. Therefore, not only is the person in the transition process, but the role may transform as well.

\section{Training Models}

The American Society of Training and Development (ASTD) has compiled a comprehensive list of best practices in Human Resources Development (HRD) to provide guidelines for the measures of behavioral change in organizational settings. Effective design and delivery is not feasible if the work environment and learners' characteristics are not considered. Learner demographic attributes include, but are not limited to job level, education, motivation, expectations, attitude, goals, and personality. Burke and Hutchins (2008) reported that the current guidelines that are considered best practices in organizational training are limited, outdated, and usually lack practicality.

A meta-analysis across various training topics consistently revealed that when training is designed and implemented with a scientific and systematic basis, it yields positive results. 
Training is a key component in building and maintaining effective leadership and workforce development. Through instruction, demonstration, practice, and feedback, individuals acquire the targeted knowledge, skills, attitudes (Salas et al., 2012) necessary for success.

Noe (2012) offered some suggestions regarding adult learning in the workplace. First, Noe recognized that employees learn best when they understand the objectives of the training. Second, employees learn better when under meaningful learning conditions, for example, when training is related to their current job experiences. Under these circumstances, training is more effective when the employees have the opportunity to practice the learned skill and implement it in a real situation. Furthermore, trainees benefit from positive feedback on specific behavior in real-time to reinforce the action and bolster their confidence for future action outside training scenarios. Modeling and observation also support trainee learning through demonstration of the desired actions.

While not all the trained competencies transfer to the workplace, Sales et al. (2012) stated that the transfer of the training is the endgame of the training programs regarding the acquisition of knowledge and skill. Transfer of learning refers to the extent to which the learned material is applied at the workplace and in job performance. In other words, training transfer represents the tangible benefits the organization receives from the training investment.

Additionally, there are pre-training, concurrent training, and post-training factors that affect the knowledge transfer, such as motivation to learn, workplace transfer climate and culture, and supervisory support of the newly learned skill sets. Beside post-training work climate, social support, self-efficacy, motivation to transfer are other influential 
factors. Martin (2010) found that peer and supervisory support both have a strong positive effect on training transfer even within a negative work environment.

Another issue to be acknowledged is that training is more than learning intervention at the time of delivery. Training personnel must consider two other periods that have an impact on learning: the pre- and post-training periods. Pre-learning intervention activities support active learning during the training sessions, and post-training follow-ups enhance the transfer of the learning into the workplace (Noe, 2012). Burke and Hutchins (2008) mentioned that the situation of the trainer, trainee, and immediate supervisor are of primary importance, while peers and the organization are the second layer of importance in the training process. Based on these factors, Burke and Hutchins proposed a training model that included some moderating variables that affect design and delivery, such as work design and type of jobs and organizational size and structure.

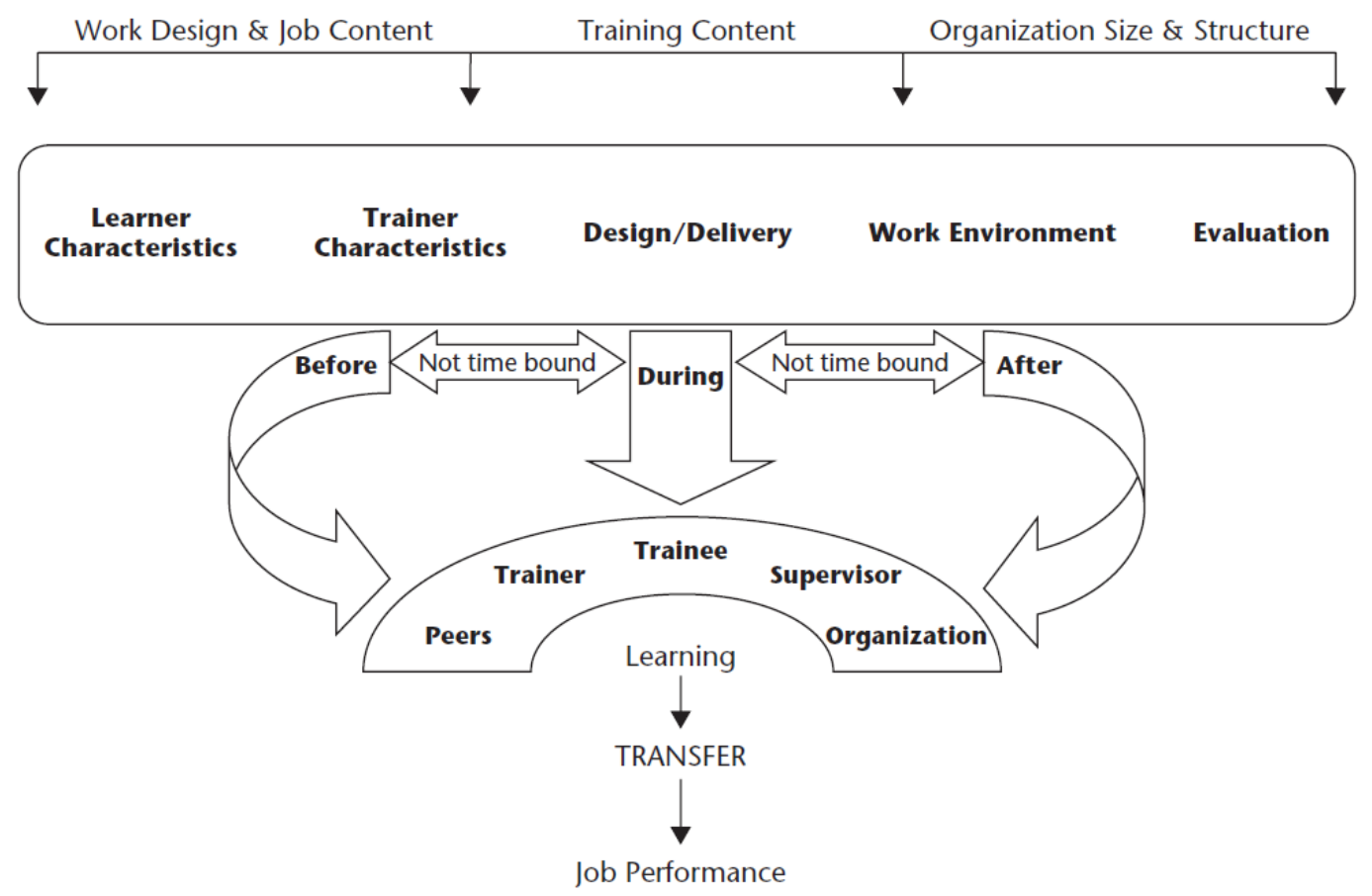

Figure 1: Model of training transfer, Adapted from Burke and Hutchins (2008) 


\section{Need Assessment}

Occupational transition often involves competency challenges in terms of skills, abilities, and knowledge for the new position (Roberts, 1994). To better understand these competency challenges, organizations conduct need assessments. The organizational or departmental analysis involves determining the appropriate training dependent on the mission of organization and the availability of the resources. Task analyses identify the knowledge, skills, and behaviors that are needed to achieve competency. Person analysis involves determining the individual deficiencies resulting from a lack of knowledge, skills, or ability to perform in performing the job.

Wentland (2003) stated training analysis happens at two levels of macro- and micro-organization. In the macro-organizational level, training analysis is aligned with the business strategy of the organization, while at the micro-organizational level, the training process places greater focus on the needs of trainees and content of the training program. In a micro-organizational analysis of the training program, four issues should be taken into account: place, product, promotion, and price. The place component may be on-the-job, such as training through job rotation, apprenticeships, and internships. It may also take place off-the-job, in formal courses, seminars, workshops, or classroom training. Yet another option can be online training courses where a trainee may decide to attend at work or at home. Product analysis is the primary question for the purpose of training. According to Wentland, the purpose is sometimes to amend the current deficiency, and others to develop skills that an organization may need in the future.

One of the first steps of the training and development process, which shapes learning outcomes, design, and delivery of training, is need analysis (Salas et al., 2012). 
At this early point in training program creation, the training program developer should recognize that training is the optimum solution for performance deficiencies and human resources issues. According to Sales et al. (2012), the three major components of training need analysis are taskjob analysis, organization analysis, and trainee analysis. In the job-task analysis, vital work functions and tasks are outlined, and competencies including knowledge, skill, and abilities are identified as cornerstones maximum job performance. This step is the blueprint for the training development. In organizational analysis, the culture, resources, limitations of the organization to support training initiatives are examined. Organizational analysis checks for strategic alignment and contextual readiness. By addressing the most pressing organizational needs through strategic analysis, resources can be relocated and deficiencies prioritized (Wentland, 2003).

\section{Leadership Training}

Leadership training programs differ from other forms of training in that trainees in leadership positions typically have more discretion in personalizing training content to fit their needs and personality style (Lord \& Hall, 2005). An effective training endeavor transfers knowledge and demonstrates desired behavior. It creates an opportunity to practice the learned knowledge and skills, and eventually should provide feedback to allow for the correction of former behaviors into more desirable ones (Salas et al., 2012). Hernez-Broome and Hughes (2004) stated that leadership competencies are the core dimension of leadership development initiatives, and the majority of leading-edge organizations define successful leadership qualities and benchmark a set of competencies. They indicated however, that the organizations should look beyond the reductionist list of idiosyncratic competencies to develop leaders.

Tannebaum (1997) reported that only $7 \%$ to $9 \%$ of skill acquisition comes from formal

training. The trends in leadership development training have shifted considerably from classroom 
teaching to development activities like coaching, mentoring, action learning, reflective journaling, and 360-degree feedback (Hernz-Broome \& Hughes, 2004). Coaching involves oneon-one learning and is usually a short-term intervention or a series of meetings over time. While mentoring is more committed long-term support of a senior member with a more junior member; in action learning, important real-time organizational problems are introduced to be solved and learned from.

Lord and Hall (2005) found that skills are learned through problem experiences, and then sets of skill are organized into a higher-level system that shape behaviors. These systems are gradually ingrained into personal identities that shape one's self-concept as a leader. Lord and Hall argued that the self-view of a leader is an essential part of developing a leadership image and it shapes knowledge acquisition, goal formation, action, and social interaction of the leader. This self-concept merges with individual identity when a novice leader desires to be seen and accepted as a leader. Naturally, they are sensitive to social feedback, and peer validation plays a critical role in their selfimage as leaders. Surface structure and behavioral leadership skills are overtly observable components of leadership where novice leaders get feedback from the reactions of the followers. In other words, these leaders develop skills by observing the behavior of other leaders and involves learning the normative behaviors perceived as acceptable to others.

Considering the lack of leadership experiences and lower levels of selfmonitoring capacities, novice leaders rely heavily on the social feedback from others when developing their leadership behaviors and habits. Ibarra (1999) stated that a combination of social, cognitive, and motivational factors leads to the adoption of a provisional identity. When exploring new roles and behaviors, a novice leader will persist 
in using behaviors that bring success, while unsuccessful ones are abandoned. These behaviors are continuously refined through social feedback from others, and it leads to solidifying one's self-view. Ibarra suggested that great leadership development is more likely in individuals who are open, exploratory and flexible about adopting provisional identities. While novice leaders tend to modify and change their own behavior based on their reaction, a more experienced leaders seeks to create change in others.

The trait perspective toward leadership has attracted considerable attention for several reasons. Traits are more stable constructs than skills. According to Lord and Hall (2005), skills are thought of as overt behavioral styles that are trainable; however, leadership is a combination of behavioral, cognitive, and social skill that may develop at different paces and styles. Developing skills also requires sustained proactivity and self-motivation on the part of the leader to hone the desired skills. These issues suggest that comprehensive leadership models must go beyond merely relying on training and development.

Thus far, I have reviewed the pertinent literature on transition of experts to the leadership and management and addressed the assessment of competency needs and training at the workplace. In the next section, I will review the department chairs' role and transition challenges.

\section{Academic Leadership Transition}

In the following section, I will focus on the transition of a faculty member to a department chair. I will briefly discuss the tasks and responsibilities of faculty and provide an overview of the faculty development programs in academia. Then, I will explain the typical duties of department chairs and the needed competencies for this position. Finally, I will review the literature on transition to department chair position and its associated challenges. 


\section{Role of a Faculty Member}

Faculty members in contemporary higher education institutions typically have three major jobs: teaching, research, and service. Finkelstein, Conley, and Schuster (2016) stated that first and foremost, the role of a professor or faculty member is teaching. However, as the role of a faculty member has evolved based on institution type, the research role can surpass faculty's other responsibilities. In these institutions, the faculty member is the main generator of information and knowledge (Gappa \& Austin 2010).

At most research universities, the typical workload expectation of faculty members is to allocate $40 \%$ of time and effort on teaching, $40 \%$ on research, and $20 \%$ to service projects (Foster, 2006). While teaching and research are self-explanatory, the service role of faculty is often vaguely defined with unclear expectations. Service entails external and internal facets with distinct differences and skill sets. These service activities advance the discipline and campus by participating in committee meetings, scheduling and organizing conferences, serving on review boards of a journal, and advising students (Palm, 2006). Internal service refers to activities in which faculty members provide support to the administration and functioning of the institution. External service functions as a means for the institution and faculty to reach stakeholders outside the higher education system to meet the public and societal needs. Faculty members usually operate beyond the campus setting, with outreach being a concept that connects the resources of higher education to the audience beyond the university campuses (Griffith, 2006). Faculty with external service assignments make themselves available as an intellectual resources by engaging with the community. 


\section{Faculty Development}

Professional development for faculty member has both broad and narrow definitions in the literature. In the broad sense, faculty development is defined as "the range of activities that institutions use to assist faculty in their roles" (Baker, 2018, p. 265). The activities may include workshops, conferences, fellowships, sabbatical, or other longitudinal programs. In a narrower definition, faculty development is assisting faculty to improve their teaching and scholarly competencies (Eble \& McKeachie, 1985). Faculty development programs are generally designed to enhance instructional development, personal, professional development, and organizational development. Professional development promotes faculty member job-related skills, knowledge, and awareness; organizational development places greater focus on developing an effective institutional atmosphere and personal development initiatives involving more interpersonal skills, wellbeing, and career planning. Bland and Risbey (2006) classified the main faculty development initiatives: leadership and management skills such as time management, conflict management, performance appraisal skill; professional academic skills such as work-life balance, navigating academic career, establishing collegial relations and networking of colleagues; organizational development such as underlying institutional norms, expectations, and values; teaching initiatives and educating the educators.

A high-performance workplace requires a culture of continuous learning to upgrade the knowledge and skills of the workforce. In the United States, the history of faculty development dates back to the earliest institutions including religious universities and colleges. At the time, institutional missions focused on maintaining the spiritual and moral aspect of the faculty, but as these missions gradually expanded, the type of faculty development broadened and evolved. Bland and Risbey (2006) mentioned that prior to the 1960s, faculty development was mostly 
limited to using sabbaticals to advance disciplinary knowledge. Until the 1960s, higher education institutions had ill-defined, non-comprehensive faculty development programs consisting mainly of orientations, conferences, and sabbaticals (Centra, 1978).

In the 1970s, faculty development programs were initially largely focused on teaching needs, typically in the form of workshops. Later, institutions added instructional, personal, and organizational development programs for faculty (Bland \& Risbey, 2006). During this period, faculty development began to take root in higher education and greater numbers of institutions began development initiatives. Alstete (2000) stated that by the end of the 1970s, most institutions reported having some faculty development programs that were mostly voluntary for faculty to attend. In the 1980s, in response to growing public concern, attention shifted to student learning and curriculum development (Bland \& Risbey, 2006), and faculty development offices across the nation started offering training through retreats, seminars, and structured workshops. Faculty development continued to become more institutionalized and more professional associations and conferences emerged (Finkelstein, Conley \& Schuster, 2016).

Gradually the emphasis on leadership, management, and administrative aspects of faculty development increased, and content areas such as organizational structure, analysis economic, political pressures on the institutions were introduced (Gappa \& Austin, 2010). More emphasis on negotiation, financial management, and performance appraisal were included in the faculty development programs (Steinert, 2000). In the 1990s, colleges and universities attempted to link faculty development to institutional mission and policies, with these programs becoming more integral, visible, and continuous. During this formative period, faculty development initiatives expanded to 
other domains such as technology, faculty wellbeing, and research productivity. Faculty vitality became inseparable from institutional vitality. Vital institutions have mechanisms in place to ensure the members stay up to date in their discipline and knowledgeable about the current advances in education, outreach, and research.

\section{Role of Department Chair}

A detailed standard job description for department chairs appears to be elusive because the duties and responsibilities vary drastically from one department to another, and chairs have the freedom to craft their role differently than those who preceded them (Aziz et al., 2005).

Tucker (1984) listed 54 tasks for the department chair in eight categories: governance, instruction, faculty affairs, student affairs, external communication, budget and resources, office management, and professional development.

Later, Carroll and Gmelch (1994) worked on the same issue, and they outlined 26 tasks for a chair in just 4 major categories: leader, scholar, faculty developer, and manager. Potgieter and Coetzee (2010) conducted a broad systemic review of competencies required of a department chair, and through a questionnaire, attempted to measure the most important skills. They proposed 18 competency modules and found leadership, financial management, and project management the most important competencies for a department chair.

According to Gmelch (1991), chairs spend 88\% less time researching and writing, $82 \%$ less in keeping up to date with their discipline, $65 \%$ less with their family, and $77 \%$ less time on leisure due to administrative duties. Overall, $80 \%$ of chairs believe that their workloads should be lightened to allow more time for research and other scholarly activities.

Wolverton et al. (1999) divided the tasks of chairs into two major categories:

administrative and leadership tasks. Department chairs are in a leadership function at three levels 
including department, faculty, and personal. At the department level, chairs have a broad responsibility for transferring the mission of the institution to the department and nurturing a supportive work atmosphere. At the faculty level, the chair responsibility shifts toward motivating faculty, increasing research capacity, improving teaching, providing more services, and evaluating the faculty members. At an individual level, the chair must acquire a survival mechanism to handle the leadership role, while simultaneously managing the day-to-day administrative functions, such as scheduling, teaching, revising curriculum, managing the budget, and managing faculty and staff.

Bowman (2002) contrasted academic chairs' function as managers while working on the structures, policies, processes, and paperwork, with their function as leaders when tackling the organizational culture, mission, and vision. Faculty members within a department desire their department chair to mainly be their supporter rather than a leader. Necessary skills and behavior of a chair fall into two categories: leadership and management, the latter of which is easier to acquire.

KSAs and competencies. Aziz et al., (2005) believe that the knowledge, skills, and abilities (KSA) approach is the most generalizable methodology to study the role of a department chair across different academic units by providing strategic analytic insight into the role of the department chair. Identifying KSAs that are crucial for training needs as a smaller number of KSAs may be targeted to address competency deficiencies. Aziz et al. (2005) believe that the KSA approach can also be used for recruitment, selection, evaluation, succession planning, and training.

Aziz et al. (2005) created a list of primary KSAs through a content analysis of the prior literature. They finalized their findings after conducting confirmatory structured 
interviews with department chairs and provided a comprehensive list of KSAs. Aziz et al. identified 79 KSAs which were rated by importance, based on the success of a chair in 10 categories: budgeting and resource allocation, curriculum and programs, department administration, external communication, internal communication, faculty affairs, legal, office management, professional development, staffing, and student affairs. Among those KSAs, the ability to bolster faculty morale and to maintain high-quality teaching, leadership skills, and knowledge of faculty recruitment, the policies and procedures are the most important.

According to HR-XML Consortium Competencies Schema (as cited in Draganidis \& Mentzas, 2006), competency is defined as "a specific, identifiable, definable, and measurable knowledge, skill, ability and/or other deployment-related characteristics (e.g., attitude, behavior, physical ability) which a human resource may possess and which is necessary for, or material to, the performance of an activity" (p. 52). Jackson and Schuler, however, essentially defined competencies as "the skills, knowledge, abilities and other characteristics that someone needs to perform a job effectively" (as cited in Draganidis \& Mentzas, 2006, p. 53).

Bryman (2007) also provided a competency-based framework and best practice guidelines for departmental leaders to improve the efficacy of chairs through training programs. Similar to other authors such as Aziz et al. (2005) and Seedorf and Gmelch (1989), Bryman (2007) claimed that his model highlights the leadership's skills that are associated with their superior performance. However, a leader's effectiveness is a nebulous concept, and some precautions are necessary to interpret these studies. Competencies and KSAs tend to be generic for many managerial and leadership positions, providing very little guidance for concrete action plans. Also, different aspects of leadership efficacy may contradict each other based on stakeholder interests. Furthermore, higher education institutions and contexts are substantially 
diverse. Hence, a comprehensive list of effective leadership behaviors may not translate across the gamut academic contexts. The notion of effectiveness can be extremely controversial, and different constituencies, (e.g., faculty members, supervisors, students, communities, staff) may have opposing points of view on the benchmarks of effectiveness of a chair (Bryman, 2007).

Department chairs are expected to function as a buffer, a facilitator, and a guide (Thomas \& Schuh, 2004). Buffering involves facilitating a clear focus, assisting faculty members to reach their full productivity earlier, and guiding them to make choices that will help them become better leaders and educators. A chair is also expected to extend this relationship to all faculty members, providing support and supervision.

In addition to meeting the expectations of faculty, chairs are expected to satisfy the expectations of the dean. The dean evaluates the chair and has a large body of constituents to serve. Chairs of a college are part of the leadership team for that particular college and work together with the dean to plan for the future direction of the college, at times putting the interests of the college ahead of their home department (Thomas \& Schuh, 2004).

Berdrow (2010) studied the role, expectations, and competencies of department chairs at a private university. Through an interview with current department chairs, she categorized her findings into four managerial tasks including faculty development, student development, communication and representation, with two additional transformational leadership tasks of catalyst/innovation and climate enhancement.

Bryman (2007) conducted a literature review study on the primary leadership behavior at the departmental level and found that successful chairs set the direction for 
the department while allowing for individual opportunities for participation and contribution in key decisions. They found that effective communication inspired trust among the faculty, and that encouragement and allocation of resources created a collegial work culture which advanced the department's credibility, both internally and externally to the university.

\section{Transitioning to Department Chair}

Professional transition and organizational socialization can be analyzed from major three dimensions. Some scholars have analyzed the transition from affective states and their associated coping mechanisms to deal with stress, anxiety, and uncertainty (Griffith, 2006; Smith \& Stewart, 1999; Willis, 2010). Other scholars center the core of their research around professional identity transformation and include studied new skills, abilities, and attributes (Aziz et al. 2005, Carroll \& Wolverton, 2004; Potgieter \& Coetzee, 2011). These researchers were interested in shedding light on competency gaps of role transition and assumed post-positivist approach to the issue. Yet other researchers investigated the behavioral outcomes and considered the cultural adaptation and socialization in the new supervisory position (Gmelch, 2004; Gmelch \& Parkay, 1999; Palm, 2006).

Affective states of transition are intertwined in both the process of competency transition and behavioral-social adaptation. Socialization is "a series of stages that an individual undergoes in making the transition from one position to another" (Smith \& Steward, 1999, p. 30). In the process of role socialization, an individual gradually understands the expected values and behavior of a position while simultaneously developing the interpersonal relationships related to the job. In other words, organizational socialization is a process in which an individual acquires the disposition required to perform a social role in the workplace. 
According to Smith and Steward (1999), workplace socialization happens in three stages: anticipation, encounter, and assimilation. During the anticipation stage, chairs have yet to be formally appointed to the position. The majority of managers have taken some form of courses or attended in-house and outside workshops on administration issues. However, most of their learning happened through self-guided activities such as reading books and articles, or though previous administrative duties, like serving on committees. Chairs mentioned that interpersonal relationships were invaluable in helping them learn more about the position before being formally appointed (Smith \& Steward, 1999). Throughout the encounter stage, the department chairs continue learning their new role after being appointed. At this point, the chairs perceive themselves being accepted by the faculty and staff, knowledge and skills are generously shared by colleagues and the former chair, and their on-the-job learning experience compensates for their initially lacking knowledge. Once in the position, informal discovery or self-directed learning are the most reported methods of learning. In this style, their interpersonal relations and skills are their biggest asset (Smith \& Steward, 1999). In the assimilation stage, chairs have accepted the role and are able to relate to the job.

Despite positive assimilation, many chairs still view themselves as outsiders: $41 \%$ of chairs left their assignment before 2 years, and $36 \%$ of chairs considered moving back to their faculty position during the first 2 years. Moreover, $63 \%$ of these chairs said that they would not recommend a chair position to others (Smith \& Stewart, 1999). Limitations of the study may have an effect on these responses. Incumbent chairs included in the study held to role for an extremely short period of time and may be related to the community college setting. Additionally, Smith and Stewart did not address 
why, despite the positive encounter stage and acceptance stages, and support of the department stakeholder, chairs did not wish to remain in the position.

Aside from the socialization, chairs experience other changes and transformations. According to Thomas and Schuh (2004), while a professor must be concerned with the students in their courses and those who are directly under their supervision, a new chair learns that all students in the department are of interest and concern; encompassing all undergraduates, graduates, majors and non-majors. This interest group population extends even to academic clubs and honorary societies sponsored by the department. While a professor may not need to know the demography of the department, it is recommended that a chair possess this information. Chairs will be in contact with students regarding complaints; from various issues involving grades to the quality of instruction, from classroom quality to virtually anything else in the department. Hence, the department chair must be familiar with the institutional procedures in place to address complaints and concerns received (Bowman, 2002).

Academic administrators enhance their credibility prior to their appointment by being part of the faculty and conducting research (Smith \& Steward, 1999). While scholarship credibility of a department chair in a research-focused context boosts the chair's acceptance among faculty, it does not directly contribute to the administrative role. However, there is expectation from faculty that the level of research performance will remain steady between chairs. (Bryman, 2007).

Gmelch and Parkay (1999) called the transition from a faculty to chair a metamorphosis. They created a taxonomy of the characteristics, which also highlight the differences between the faculty and chair roles, to describe how chairs adjust during their transition period: 
- College professors spend most of their time working alone even when they are coauthoring and collaborating, while a department chair position requires constantly working with others: from solitary to social.

- Professors work for uninterrupted periods, with the ability to focus on their scholarly pursuits, while departmental leadership involves many short and fragmented endeavors: from autonomy and autonomy to accountability and fragmented work periods.

- $\quad$ On the one hand, professors, by and large, have flexible working conditions; from location to time and generally have a large degree of autonomy regarding activities. Chairs, on the other hand, do not have the same level of independence and are directly accountable to their supervisors, faculty and students.

- $\quad$ Professors are able to spend time writing scholarly manuscripts, while chairs must learn the art of persuasion and precision through memos: from manuscripts to memoranda.

- Professors may block out longer periods for their scholarly work and in contrast, chairs are obligated to be accessible: from closed to open door policy.

- While professors generate knowledge, chairs essentially practice the art of compromise and persuasion: from profession to persuading.

- Professors experience more stability in their career and retain their professional identity. Meanwhile, chairs and administrators are more mobile in higher education settings: from stability to mobility.

- Professors request and expect resources from their university, and chairs act as a custodian and manager of the same resources: from client to custodian. 
- It is perceived that chairs have higher compensation and benefits from their positions. while the difference is insignificant: austerity to prosperity.

This 9-branch taxonomy illustrates how the stable and relatively predictable life of a faculty members transforms, and suddenly many new responsibilities are thrust upon them. As Hecht (2001) asserted, chairs find themselves "operating in a murky landscape of either illdefined or conflicting expectations" (p. 7).

\section{Challenges of Department Chairs}

Most new department chairs have two attributes in common; they have achieved success in the promotion and tenure process but have little formal preparation for an administrative position (Thomas \& Schuh, 2004). Considering the range of responsibilities of a department chair has for its constituents, it is shocking that many higher education institutions provide minimal formal training or preparation programs for the appointed chairs, given that many of them have no prior administrative experience (Aziz et al., 2005; Bryman, 2007; Seedrof \& Gmelch, 1989).

Carroll and Wolverton (2004) suggested that "dealing with people, coping with the bureaucracy of the institution, and the negative impact on research productivity" (p. 7) are the three major stress-generating categories for a department chair. Chairs engage in various managerial conflicts and must meet deadlines and impose deadlines on others. They must take charge of department financial matters, and many times do not have clear guidelines or training for these duties. Often, they cannot determine how they are evaluated by supervisors and feel a general lack of efficiency in their tasks (Carroll \& Wolverton, 2004). Managerial stressors including program approval, complying with rules, resolving collegial issues led to an additional $20 \%$ more stress for chairs compared with faculty members (Gmelch, 1991). In compensation for 
the above-mentioned issues, Tucker (1984) stated that department chairs receive an administrative stipend, with the position perceived as having greater prestige. Also, they may gain personal satisfaction from being influential and being able to lead and help others.

One challenge of the transition to the chair position is the new relationship dynamic with old coequal colleagues (Thomas \& Schuh, 2004). In the new position, the chair is now in charge of the department and in an academic and administrative leadership role that makes decisions that affect formerly equal colleagues. This level of authority has the potential to create friction within the department while the quality of the relationship and the nature of their friendships also undergo scrutiny by all parties (Griffith, 2006). The new administrator must become thick-skinned enough to bear the constant pressure and criticism (Willis, 2010). This think skin aids department chairs when confronted with situations that come in conflict with their other roles and with their value system as a former faculty member (Palm, 2006). Making decisions that serve both the faculty and the higher administration can be quite a challenge. Especially during the initial stages, role ambiguity is not uncommon since the roles and associated values are not clearly articulated in terms of expected behavior or actions (Gmelch \& Parkay, 1999).

It is interesting that after learning the ropes in the position, only one in five chairs choose to advance their careers into other administrative roles (Tucker, 1984) and, on average, department chairs serve for approximately 6 years (Thomas \& Schuh, 2004). In the next section, I will review the literature on the transition process of faculty into a department chair position. 


\section{Theoretical Framework}

In this section, I will present the theoretical framework, and explain how this framework properly fits this study. After reviewing the transition theory of Schlossberg (1981, 1995, 2011), I discuss the various factors that affect professional transitions.

\section{Transition theory of Schlossberg and Types of Transitions}

Schlossberg (1995) categorized transitions into anticipated, unanticipated, or non-event transition. Anticipated transition happens predictably and usually allows the individual with time and opportunity to prepare. These expected events include graduation, marriage, retiring, etc. As the name implies, unanticipated transitions are unscheduled events and were not usually predictable transitions. Pearling (1980) states that unanticipated events involve crises and disruptive conditions such as, being fired, laid off, an unexpected promotion, divorce, the death of a partner. Generally, their probability of occurrence is low. Non-event transitions are the ones that are but did not happen, hence altering the individuals' life, such as a promotion having never been offered, a marriage that did not happen, a child that was not born. Schlossberg (1995) clearly stated that an event or non-event for one person can have a different meaning for another and the type and nature of transitions vary from one case to another case.

While the onset of transition may be marked by a singular event/non-event, the process of transition takes time and evolves with the individuals' changing reaction. People gradually begin to distance themselves from past roles and conditions, oscillating between the two, before ultimately acclimating to their new role. (Schlossberg, 2011). Bridges (2004) stated that the transition's onset is an ending where the individual disengages, disidentifies, disenchants oneself from old roles and routines. This phase is the prelude to the next stage which is a neutral zone. The neutral zone is a time-out period from daily routines and roles and meant to be a moratorium 
for the person undergoing transition. According to Bridges, transition phases end with the beginning when a person leaves the previous phases and accepts the new roles and routines.

An individual's appraisal of the transition is critical. Schlossberg (2011) believed when an event happens, the impact of the event on the individual's life, relationship, routines, assumptions, and roles is vital in studying the transition. The context also shapes how individuals react to transition. It can be everyday life strain or issues regarding occupation, marriage and relationships, family, and/or mental or physical health. The transition can be initiated with the individual or with someone else. The transition may be personal, interpersonal, or it may involve the public and one's community. These conditions and many more affect the transition of the individual. For instance, if it is personal, the resources can be mobilized, and support can ameliorate the impact of a negative transitional shock.

Transition varies based on the situation that occurs. The individual's perception, and whether they consider it positive, negative, or insignificant, shapes the coping mechanisms during the transition (Lazarus \& Folkman, 1985). Peoples' sensitivity and vulnerability, as well as their interpretation, affect how they experience the change. Understanding the many factors affecting transition helps the researchers better understand the transition process.

Schlossberg (2011) Transition Model spearheaded the movement toward understanding and coping with transitions. This model answers the questions of how one handles a transition journey and how do they live through and learn from it. She proposed features common to all transitions even if each transition is dissimilar to others. These features, which may be potential resources or deficits, are clustered into four main groups of self, situation, supports, strategies. 
Self, according to Schlossberg (2011) refers to the individual's inner abilities, personality attributes and attitudes. For instance, an optimistic person will positively interpret future uncertainties, and with the power of optimism they are able to move forward and survive. While another individual may experience a different quality of transition due to their own disposition. Situation refers to the circumstances of the person at the time of transition. For instance, there are other stressors at the time of transition must be considered.

In addition to self and situation, support available to the individual is critical in the quality of transition and individual's well-being. Imagine, if the individual who is moving to a new city and knows no one, this circumstance will make their transition and adaptation more difficult than the person who already has family and friends in the new place. Professional associations can be a source of support for someone who is going through a job transition making the navigation of challenges easier. Strategies, as defined by Schlossberg (2011), are those coping initiatives that change the situation, reframe the situation, or prompt the individual to seek support. In other words, a person in transition strategies asks themselves multiple questions: Are there ways to change the situation? If not, are there other ways to look at the situation? How can I ask for help or seek support? Overall, these four variables can be assets or liabilities and resources or deficits of the individual who is going through a transition. This transition model of self, situation, support, strategies provides the structure to study and analyze transitions.

\section{Summary}

This review of the literature serves as an introduction to the transition of the faculty members to administrative and leadership roles. In the interest of narrowing scope of the study, I have addressed the overarching issues of transition of field experts to the leadership and 
discussed various career development theories and leadership training schools. I later explained the role of faculty and expectations of department chairs. However, gaps in information exist regarding empirical research on the challenges of transition and skill competencies of department chairs. The next chapter provides a comprehensive overview of how a qualitative case study best supports the purpose of this study and leads to significant findings with implications for research, practice, and theory. 


\section{CHAPTER 3: RESEARCH DESIGN AND METHODOLOGY}

The purpose of this research is to elucidate the faculty experiences and perceptions, and to make sense of their transition processes into department chair. I approached this study from a qualitative perspective to gain an in-depth understanding of the experience of transition, its complexities and navigation of its challenges. The case study is bounded analysis of the real-life transition experience of chairs at University of Missouri. In this research, I address two research questions:

1. How do new department chairs experience the transition from a faculty position to a leadership role?

2. How do new department chairs navigate the challenges of the transitioning to leadership? This chapter will cover the epistemology, methodology, positionality and limitation of this study.

\section{Constructivist Epistemology}

My epistemological positioning as a researcher has shaped my approach to this study. I approached this case study from a constructivist point of view to delve into the sense making process of the faculty in their transition into department chairs. From a constructivist's standpoint, the truth is relative and dependent on one's perspective. I intended to reconstruct the realities that my interviewees created while acknowledging multiple interpretations of their transition processes. Baxter and Jack (2008) asserted that one advantage of the constructivist approach in a case study is the close interaction between the researcher and participants enabling the vulnerability necessary to disclose their experience and share their stories. In this process, the researcher observes the reality from the participant's point of view and can better understand their lived experiences. Constructivism considers the sense making of each person valid and worthy, acknowledging the unique experience of each individual (Patton, 2015). 
Constructivism allowed me to study how new chairs perceive the challenges of the transition and how they navigate these challenges in various circumstances by acknowledging the subjective nature of sense making in the experience and construction of their perceived reality. Constructivism suggests that knowledge is the aggregation of human-made constructions; hence, reality an internal construction, where meaning is assigned to experiences and ideas. Savin-Baden and Major (2012) believe that constructivism fundamentally is the notion that reality is in the minds of people who construct it, and knowledge is not independent of the knower of it. Researchers who adopt this approach are attempting to gain data about how individuals construct their knowledge and make sense of their experience. In the constructivist paradigm, "Phenomena can only be understood within the context in which they are studied; findings from one context cannot be generalized to another" (Patton, 2014, pp. 124-125).

A constructivist paradigm, in my opinion, meshes well with a qualitative case study design to explore the complexity of the transitions of a department chair. This paradigm allows me to consider the uniqueness of their experience. Constructivism allows me to study the role of context in shaping the subjective reality of new chairs during the transition phase. In the next section, I will explain the methodology and design of this research.

\section{Methodology and Research Design}

I conducted this study using qualitative methodology as it allowed me to search for the entire experience of the transition. Qualitative research is an interpretive-naturalistic approach to phenomena, or problems, in their natural settings in order to make sense of and interpret them (Denzin \& Lincoln, 2002). Merriam (1998) stated that "qualitative researchers are interested in understanding the meaning people have constructed, that is, how they make sense of their world and the experiences they have in the world" (p.6). This methodology enabled me to gain an in- 
depth understanding of a complex issue that is intertwined with multiple factors so I can study how chairs navigate this transition. I explored the professional life, lived experience and the department chairs' shared stories; ergo, utilizing qualitative methods of inquiry allowed to unveil this experience in its context and from a holistic perspective (Patton, 2015).

My other rationale for choosing this approach is that existing literature on the transition process of a faculty to a department chair is in its early stages and still in the exploratory phase (Baker et al., 2018). The best practices of department chairs, their succession procedures, and training and development programs for this level of higher education leadership are not well defined (Berdrow, 2010). Because quantitative methodologies seek to quantify the data and use standardized questions and predetermined response options, qualitative methodology is more suitable when additional information on an area is desired (Patton, 2014). I believe, gaining a more in-depth understanding of the nature of this experience and its associated challenges contributes substantially to the present body of knowledge on academic leadership.

Merriam (2009) stated that qualitative researchers try to understand how people construct and make sense of their world. This study had major characteristics that Merriam has listed for a qualitative research which are: focus is on the process and understanding the meaning, and the researcher is the instrument of data collection and analysis. I interviewed and interpreted the participant responses and derived meaning from them. Through an inductive process, I reached an outcome that is richly descriptive. The goal of my research approach was to reconstruct the reality that department chairs have experienced in their transition period and the challenges they have endured. To depict the reality of the transition that chairs construct in this process, I provided a rich description of the transition sense making through an embedded single case study design. In the following, I provide more detail about how I have designed the case study. 


\section{Case Study Design}

Case study design enabled me to understand the real-life transition experience of the faculty members at the University of Missouri, and how they are encompassed in the broader context of their institution as well as the more salient contextual conditions of their department. According to Merriam (1998), the case study is an "intensive, holistic description and analysis of a bounded phenomenon such as a program, an institution, a person, a process or a social unit" ( $\mathrm{p}$. xiii). Yin (2013) defines a case study as "an empirical inquiry that investigates a contemporary phenomenon in depth and within its real-life context, especially when the boundaries between phenomenon a context are not evident" (p. 18). Case studies allow in-depth exploration of a bounded system within its context to understand the particularity and complexities of a single case in its context and within its important circumstances (Creswell, 2013; Stake, 1995).

The end-product of a case study should be particularistic, descriptive, and heuristic (Merriam, 1998). I tried to be particularistic by focusing on a particular group, i.e., department chairs, in a particular situation, i.e., the transition period in their department. I am descriptive with providing rich, in-depth descriptions of the phenomenon. Furthermore, I created this study heuristically by practically illuminating the phenomenon and reconstructing the reality of transition of the chairs.

Stake (1995) also suggested that case study research is the preferred method due to the degree of control the researcher has on the phenomena. In this study, I exert no control or authority over the transition of faculties into department chair positions. I also have no vested interest, nor is it feasible for me, as a Ph.D. student, to manipulate or control their transition in any form. Unlike experimental methods where investigators can manipulate behaviors directly and study the variables, or the quasi-experimental designs that use the logic of randomization 
and treatment, the case study research method does not need control of the investigator over the subject of the study.

Two major characteristics of case study research are that it defines the topics broadly rather than narrowly and tend to cover contextual multivariate conditions rather than isolated ones (Yin, 2013). Case studies are not particularly defined on any variable, rather it is defined on context. Each participant is considered a separate unit of analysis that helps understand the phenomenon of the transition of a faculty member to departmental leadership position at the University of Missouri.

Baxter and Jack (2008) recommended that a case should be bound by time and place, time and activity, and definition and context. Regarding time and place, this case study focuses on the transition period of faculty members at the University of Missouri. For the purpose of this study, the transition period will be defined as the first 2 years and will investigate the transition and the lived experience of chairs. Regarding bounding the definition and context, I have defined transition in the previous chapter, and in the next section, I will discuss the boundary of the context of this study.

I chose a single embedded case design for this research. Unlike the holistic design that examines the global nature of the case, single embedded case designs allow for more specific understanding of the phenomenon in operational detail (Merriam, 2009). I investigated the transition of multiple participants to reach greater understanding of department chairs roles. All cases take place at the University of Missouri. One reason I chose multiple units of analysis was to incorporate multiple angles to analyze the issue; these different views gave significant opportunities for extensive analysis and enhanced insight into the case (Stake, 1995). In this 
section, I have explained why I have chosen embedded case study to explore the topic. In the next section, I will address the epistemological orientation of this study.

\section{Research Context}

Familiarization with the context of research is of paramount importance in case studies. Since all the participants are selected from the University of Missouri (MU), here I attempted to provide essential information about the university. The context of this case study is the University of Missouri-Columbia, which is a public land-grant university of the state of Missouri with a high undergraduate population. With its more than 300 degree-programs through 19 colleges and schools, MU is the state's most comprehensive university. Endowment funds totaling approximately $\$ 1$ billion, MU had more than 30,000 enrolled students and 13,356 fulltime employees and 2,129 faculty instructors in the academic year 2018-2019 that this study is conducted. (Missouri.edu, 2018).

The University of Missouri is often considered a flagship university. It is a member of Southeastern Conference (SEC), which participates in the Division I of National Collegiate Athletic Association's (NCAA). Based on the Carnegie classification of institutions of higher education, this institution is recognized as a large, public university, and it is classified as an R1, or very high research activity university (Carnegie, 2018). The University of Missouri is also a member of the Association of American University (AAU).

Based on the Collective Rules of the University of Missouri (University of Missouri System, n.d.), departmental leadership is decentralized on this campus, and department chairs are bestowed with a large degree of autonomy. At the University of Missouri, the chair is the chief executive and academic officer of the department. Within the rules and boundaries of the department, the chair is responsible for the effective and efficient administration. In this 
institution, chairs are appointed by respective chancellors upon recommendation by the dean of the school or college, and these appointments are subject to periodic reviews by faculty and deans (University of Missouri, n.d.). The Collected Rules of the University of Missouri states that chairs are in charge of fiscal, staff, teaching, students and other miscellaneous duties in the department.

\section{Participants and Selection Procedures}

There are several criteria for participant selection for the purpose of this study. The participants must be first-time department chairs at the University of Missouri who are in transition process. I reached out to each college and school, with exception of the School of Medicine, School of Nursing, School of Health Professions, the College of Veterinary Medicine. My rationale for excluding these schools is twofold: first, healthcare-related schools have a different set up and function on campus due to their didactic, professional, and clinical trainings. Second, since I have had a minimal exposure to these fields, I, as the researcher, have a more indepth understandings in other disciplines. The participants were 3 associate professors and 4 full professors. Regarding their fields, 2 of them were in Science, technology, engineering and mathematics (STEM) majors, 2 in social sciences, 3 in humanities. 3 of the chairs had limitation of only one 5-year term of leadership and 4 other ones, 2 terms of 3 years.

According to Howard (2003), the transition process begins when the managerial tasks occupies more than $50 \%$ of the individual's time, and this transition period ends after 2 years of formal appointment in the role. For the purpose of this study, the transition period is defined as the first 2 years in the position of department chair. The department chairs in their first two years are in transition because the first year would be the first time, they are responsible for academic 
leadership duties, and in the second year, they are still in process of learning and adopting to the new role (Howard, 2003).

I created a list of all the chairs on campus. I sent an email to the chairs and invited the chairs who are in the first two years to participate in this study. I sent the invitation email to the potential participants to introduce the study and request two sessions of one-hour, face-to-face meetings. For this case study, I recruited seven participants. This number of participants provided sufficient variation and depth of data for a case study. I also selected the participants purposefully to insure maximum variation in terms of gender, race, discipline, and years of experience as faculty. The goal was to not bias my selection toward one particular gender, race, years of experience, or faculty rank.

I emailed the consent form 2 weeks prior the interview date, and I provided a printed format on the day of interview to attain their signature. I also got direction from University of Missouri's Institutional Review Board (IRB) regarding consent form creation. The consent form (see Appendix II) included the right of voluntarily participation including withdrawal from the study at any point; purpose and the procedures of the research; the length, risks, and rights of participants; confidentiality and privacy issues and possible benefits; dissemination and publication related information; my contact information; and signatures of participants and the researcher. To further protect the rights of my participants, I actively protected their identity and privacy by restoring all the data in password protected machine with a second layer of encryption codes on each file. I also anonymized the participants identity by assigning a pseudonym to them and removing the identifying data from the extracted information. Finally, the interview purpose and general interview topic was giving to them to reflect on their responses at the interview. 


\section{Data Collection}

The adequacy and appropriateness of the research method depends on the purpose of the study and the research questions (Patton, 2015). I collected various types of data for this study. At the core lies two rounds of interviews with the chairs which I describe in detail in the next section. I also collected other documents that are listed below. While these documents are great sources of information to understand other facet of transition, I used them to triangulate the interview findings. As Bowen (2009) stated, documents are an unobtrusive and non-reactive form of data collection that are not affected by the research process or the presence of the researcher.

Interviews. According to Seidman (2013), when the researcher is seeking subjective understanding and sense making of an experience, interviewing is an appropriate avenue for this type of inquiry. The interview method enables researchers to probe topics and issues, and it helps to understand how a participant in the study formulates his or her world (Seidman, 2013). This case study design puts particular importance on the subjective nature of the transition experience and the role of context and individual differences shaping this reality. The interviews enabled me to gain access to complex, in-depth information about chairs' transition processes, challenges, and how they navigated this experience.

My goal in conducting the interviews was to build upon and explore the research questions, having the participants reconstruct their experiences during their transition period and the way they understand their experience. According to Seidman (2013) this sense making phase requires the participants to look at how various factors interact with each other to create that experience for them. Their present understanding of what happened or is happening in the context is of paramount importance at this point. It is a combination of exploring the past and 
reflecting on it. Furthermore, Patton (2015) mentioned interviewing as an effective method to discover what cannot be directly observed and is a way to gain in-depth knowledge and understanding of a phenomenon. Additionally, interviewing is a suitable method to collect data when the researcher needs to probe deeply into participant experiences. I created the interviews to be meaningful and understandable by conducting them in the context of their lives and influential factors affecting their transition. Seidman (2013) points out that without context, the possibility of exploring the meaning of an experience is little.

I utilized the semi-structured, open-ended interview style, which allowed sufficient flexibility to explore responses based on emergent needs and inputs during the interview process and invited the participants to share their experiences through stories and opinions. It also allowed me to go off-script to explore developing themes and have a more personalized conversation with the interviewees about their experiences. Savin-Badena and Major (2012) believe that semi-structured interview has the advantage of interaction being more focused and purposeful, while open-ended questions allow interviewees to express their ideas and enable the researcher to compare data on the same topic or issue.

As Patton (2015) asserted, for a case study design, collecting data in interviewee's context is vital since what distinguishes a case study from other research designs is the importance of the context on the units of analysis. Two rounds of interviews were needed to collect data to address the two research questions and cover all the questions of the interview protocols. In the first interview my focus was on covering the first research question, thus, I gathered data on the contextual factors of the transition. In the second interview, I clarified if there is any issue with first research question and I addressed the second research question. I conducted the first interview with all participants before going to the second interview. I adjusted 
the protocol for second round of interviews based on the first interview protocol and after preliminary data analysis. I scheduled the second interview approximately four weeks after the first interview to have sufficient time for reflection and primary analysis.

Prior to conducting interviews with the participants, I conducted pilot interviews to gain an additional understanding of the concepts before further refining the interview protocol. I received feedback from my research dissertation committee on my interview protocol and techniques. Conducting this pilot study helped me to discover the potential flaws and provide the opportunity to revisit the design of study and understand its challenges. It also provided the raw data for initial data coding and critical analysis of the process to probe future limitations.

The interviews were conducted face-to-face in the department chair's office, and they lasted approximately 60 minutes. In the next section, interview protocol, I will describe each interview session.

Interview protocol. The interview protocol addresses the research questions and provides direction to follow during the interviews (Patton, 2015). I created the interview protocol by putting research questions at the center and looking at it from the theoretical framework. In other words, I investigated how the new department chairs experienced the transition from a faculty position to an administrative role, and how they navigated the challenges of the transition. Below, I will explain how I framed the protocol from a constructivist point of view, and how transition theory shaped the questions of the protocol.

Since the interview protocol for this case study was developed based on Schlossberg's transition theory $(1981,1995,2011)$, I formulated the questions based on four major features of the transition model, which are situation, self, support, and strategy. In line with constructivist premises, I explored the transition from their point of view, and led the interviews with open 
ended questions to provide a space where the participants can portray the journey that they have gone through.

In the first interview, I established rapport with chairs and reconstructed their context to understand their professional background, with my main goal to dissect the transition experience of the chairs and the challenges that they faced. I put the participants' experience in context by questioning to discover more about the participants in light of the topic. I focused on the self and situation parameters of Schlossberg $(1981,1995,2011)$ and concentrated on the participants' experience in the transition while striving to reconstruct the interviewees experience in the transition phase.

In the second interview, I focused my questions on the support and strategy constructs of Schlossberg. In this way, before delving deeper, I started with establishing an understanding of the individuals and their circumstances and the experience of how they navigate and solve problems. I sought out information that was not discussed in the first interview and I asked the participants to reflect on the meaning of their experience and how they are dealing with the challenges. Through these semi-structured interviews, I sought the same information across the board, and with the follow-up questions could address the idiosyncrasies and differences among participants.

It is important for me to collect the data face-to-face in their natural setting to build a stronger rapport with the participants and gain their trust; that may not have been possible via a phone interview. In order to ensure the confidentiality of the participants, I assigned them pseudonyms. Only the researcher has the access to the identity of participants. After gaining participants' consent, the interviews were recorded by a digital voice recorder. 
Curriculum vitae. My goal of collecting their Curriculum Vitae (CV) was to understand their educational and professional background. The information from the $\mathrm{CV}$ allowed more informed interviews. The commonalities and differences among their careers, transitions, academic fields and expertise were valuable in coding their interviews. The faculty's curriculum vitae revealed some aspects of the faculty members' academic background and interest. But most importantly, they gave me information on the career path and, to some extent, the career trajectory. I learned about their previous career transitions, and I saw the leadership and managerial roles and duties undertaken to this point.

Calendar. I asked for a typical 2- week calendar from the participants. The calendar was a great resource to tap into the daily life of department chairs and their time allocation to different activities. In conjunction with other sources, it helped to portray a more encompassing picture of professional experience of department chairs and better understand their daily life. I requested for participants in the second interview, to open their last two weeks calendar and briefly tell me all their meetings and activities for each day. I also asked them to provide information about the type, frequency and the nature of the meeting. I made clear to them that it was not necessary to name the individuals they are meeting.

Appointment letter. Through these documents, I tapped into the expected job responsibilities, description, workload distributions, expectations, commitments and contingencies are presented in the letter. Being cognizant of the expected qualifications and requirements helped me understand the nature of the job better. I requested this document from the department chairs. 


\section{Data Analysis}

According to Stake (2006), data analysis does not start at any particular moment. Merriam (1998) suggested to analyze qualitative data simultaneously with data collection. I started coding my data while collecting the data to gain an immediate insight into the topic and if there became a need, I could change the direction of the study. As mentioned in the previous section, the theoretical framework not only guided me in the data collection and protocol design, it shaped the data analysis and contextualization of the findings. The collected data were analyzed into two broad categories, interview data analysis and document data review and analysis. I then imported these data to NVivo software to manage and code them.

Interview data analysis. After conducting the interviews, I transcribed the records to start the process of converting the raw data into meaningful information. With respect to the conceptual framework and research questions, I analyzed the data to inductively explore the patterns and themes across collected data. I followed the guideline of Patton (2015) in criterion of themes and categories by looking for recurring items and sorting them into themes, and I constantly re-examined these themes based on internal homogeneity and external heterogeneity thorough out the process. In other words, each theme included everything in detail and exclude any unrelated data. The overall objective of the analysis was to discover patterns in the data. I tried to "see patterns, identify themes, discover relationships, develop explanations, make interpretations, mount critiques, or generate theories" (Hatch, 2001, p. 148). I also remained reflexive throughout the entire process and reflected on the interviews and documents at all stages of the data collection and analysis.

After doing the initial coding of the interview transcriptions, I continued the coding process into subthemes. Patton (2015) indicated that qualitative analysis is the continual process 
of analysis and coding of information, so I returned to the first layer or primary codes and themes several times and started the process with the new set of codes, themes, categories, and patterns that better fit the data and purpose. I explored the emerging codes and themes, and searched for explanations and alternatives. The ultimate goal of these analyses was to transform the raw data into useful findings.

Document analysis. Document analysis is a systematic procedure for reviewing and analyzing documents "to elicit meaning, gain understanding, and develop empirical knowledge" (Bowen, 2009, p. 27). Document analysis involve skimming, examination of the data and reading and categorizing the documents and their interpretations. It is an iterative process that combines premises of content analysis and thematic analysis. Via content analysis, the data are organized into categories based on the central questions of the researcher. And in the thematic analysis, patterns were recognized and coded within the data in a process of re-reading and reviewing the documents (Bowen, 2009). I coded the collected documents into into various categories and themes in conjunction with the interview I interpreted them.

\section{Positionality}

The researcher plays a pivotal role in all stages of qualitative research. As Merriam (2009) stated, the researcher is an active ingredient and a key instrument in data collection and data analysis. Hence, discussing the positionality of the researcher is crucial to establishing credibility for the findings of this study. While a part of my positionality has been implicitly revealed so far through the epistemology, design, and methodology, in this section, I bluntly acknowledge my biases and position.

I was born on the border of two countries, Iran and Turkmenistan. While I am ethnically a Turkmen, my nationality is Iranian. I was raised fully bilingual and multicultural. In my early 
ages, I noticed that perceived realities of the people surrounding me were fluid and ambivalent. The way my grandmother, a monolingual Turkmen, portrayed the world was very different from my monolingual Persian teacher. She fascinated me in my young ages with metaphysical and magical reasonings while the Persian version of the reality did not sound anything like that. Growing up, I noticed that some people unanimously in a culture used to call an event the consequence of an action; while for other group of people, that same event was the way of God to test a man, or some sort of divine interference.

I learned that norms and expectations are drastically different in different settings, and for me to conform to societal expectations, I had to switch constantly from one worldview and to another. I was moving between universes; this allowed me to see how fluid realities are and how ambivalent everything is. I lived a life of multiple mindwares and multiple cultural reality tunnels, and I learned people are living different realities and each perceives and produces one different from the other. And I learned these realities conflict and overlap. All these experiences at a young age made me open to connecting the dots in a new way. I became more open to different worldviews. I learned that reality is tightly coupled with perception, and perception is tightly coupled with culture and culture to language. I realized that all of us are, somehow, living in different universes. This epistemological stance is very much in line with the premises of constructivism that people arrive at their own version of the truth which is influenced by their unique background and embedded worldview.

Regarding the positionality for the purpose of this study, another pivotal point is my academic and professional background. I got my bachelor's degree in English literature. Following that, I began a graduate degree in teaching methodology. Before finishing my second semester with this program, I decided to do an MBA simultaneously as I noticed many 
educational issues in classrooms are not method problems but at some level administrative and managerial.

I worked in the human resources development department of a petrochemical complex. I witnessed how engineers transitioned into new roles, and how they tried to adapt and learn. I learned about these formally and informally via multiple channels, through face-to-face interactions with the people who were experiencing it, their supervisors and peers, and formal evaluation and appraisal tools. The transitions varied in nature: relocating, changing projects and task type, changing roles horizontally, earning a promotion, and so on and so forth. It always amused me to learn how individuals deal with the transition.

Here at the University of Missouri, while pursuing my Ph.D. in Educational Leadership and Policy Analysis, I have worked on various assistantships and grant projects. I gained much experience in qualitative research. I have accumulated experience interviewing and analyzing interview data in two grant projects where for I worked more than 18 months. I developed my research acumen by interviewing 12 educational administrators in one grant project and assisting with developing code and analyzing more than 30 transcripts in the other. Outside of these two grant projects, for one semester I worked as a graduate research assistant interviewing master's student of Educational Leadership and Policy Analysis who had assistantship position on campus to learn about their professional and academic experience at the University of Missouri.

I continued to enrich my understanding of faculty development by working for the Faculty Institute for Inclusive Teaching (FIIT), where inclusive pedagogies are promoted to MU faculty. Being involved with this program exposed me to faculty development initiatives and their challenges, which was a tremendous help in understanding faculty development and potential challenges of the transition into the department chair position. 


\section{Trustworthiness}

I used various means to ensure the trustworthiness of this study, and simplified it to four criteria of confirmability, dependability, credibility, and transferability (Lincoln \& Guba, 1985). Triangulating data, conducting a pilot study, peer debriding, creating an audit trail, and providing in-depth description are the major means to achieve trustworthiness. In the following sections, I will elaborate on how I am planning to address each criterion.

\section{Credibility and Dependability}

Credibility is the truth and accuracy of the finding (Lincoln \& Guba, 1985). I voice recorded and used verbatim transcription to establish referential adequacy that Lincoln and Guba (1985) suggested use to enhance the credibility of the study. Interviewing multiple participants made the data more credible by eliminating the possibility of unintended and uncontextualized biases of collecting the data for the case from one participant.

To further enhance the credibility of the study, I utilized my dissertation committee to review the methods and coding process. Among the committee, are experts in qualitative studies and others with experience in the transition to the chair position. This review further supported the decision regarding the method choices and enhanced the credibility of the research. Since I transcribed the interviews and coded the data after completing the first round interviews with all participants, I discussed the emerging themes and ambiguities in the second round to clarify and reconstruct their transition process. This member check is another technique, that ensures the credibility of the findings.

My 5 years of experience at the University of Missouri studying higher education administration gave me satisfactory understanding of the culture, context, and setting for the scope of the study. Familiarity with the context of this case study enhanced the credibility as this 
exposure enabled me to better understand the experience that department chairs have gone through.

Despite using multiple researchers, utilizing various frameworks and theories, in addition to a combination of various methods and data sources in the study, one the same difficulties encountered is the means of triangulation. Bowen (2009) suggested that confluence of evidence from various sources breeds credibility. For this purpose, I used interviews and various documents. The convergence and corroboration of these different data sources and methods reduced the impact of potential biases that can result from a single data source.

Dependability is another criterion that must be addressed to ensure trustworthiness. According to Lincoln and Guba (1985), dependability is a question of replicability and consistency; in other words, a dependable study is one that findings of the inquiry can be hypothetically replicated under the same condition. I kept all the memos, briefings, summaries, and pertinent notes before and after interviews. I also reported a transparent description of each research step from the beginning through development and reporting. By creating this audit trail, I assured dependability and conformability of the study (Lincoln \& Guba, 1985). In the coding process, I analyzed the data from multiple different angles. I triangulated the findings with the findings of similar studies to confirm or provide alternate explanations for the differences and incongruencies.

\section{Confirmability and Transferability}

While the researcher cannot ever reach absolute objectivity, according to Lincoln and Guba (1985), confirmability is the extent to which findings are the result of the experience and idea of participants rather than researcher's biases and position. I have already stated my positionality and biases, which shaped my interpretation of the study data and helped to boost the 
comfortability of this study. Marrow (2005) indicated that procedures to establish credibility share plenty of techniques with the other trustworthiness elements such as triangulation.

Furthermore, I sought peer debriefing with my dissertation committee members who are familiar with qualitative analysis of data to receive feedback throughout data collection, analysis and reporting. I stated my positionality and biases that shape my interpretation of the study data.

To increase the transferability of the study, I included a wider range of discipline and departmental variation at the University of Missouri. A detailed description including contextual references of the participants experiences also enhanced the transferability of the findings. Furthermore, I included more detail about the context and nuances of each individual chair to avoid the overgeneralization of the situation to impertinent ones.

\section{Methodological Limitations}

All studies have limitations regardless of their sample size, diversity of sample and methodology. However, it is important to recognize and acknowledge the limitations of the study and design to reduce them as much as possible. This study has some methodological limitations that restrict the implementation of the study.

First, the case study designs are inherently prone to issues of validity (Creswell, 2014), and this research is no exception. Case studies are usually limited to a certain number of participants with a circumstance. It makes it difficult to reach to the level that covers a wide range of voices in various situations. This case study is limited to a single institution, the University of Missouri at Columbia. The ecosystem, leadership, climate, and context of the campus play salient roles in academic leadership experience. The findings will be limited to the situations that are similar to this campus and may not be transferrable to other institutions with considerable contextual differences. In addition, the individuals who agreed to be interviewed 
may not be representative of all the department chair of this institution. This study was limited to chairs in seven departments from in campus of more than 100 departments.

The design relied heavily on interviews and what participants chose to share as their experience, thoughts, and perceptions. I essentially relied on their recollections on their experience and understanding of the transition. This made the study prone to memory deterioration in the out-of-transition chairs with potential lack of perspective or being too close to the issue with in-transition chairs. Campus and departmental politics, confidentiality, hesitations, and many other factors may also lead to selective disclosure of the experience. This study is vulnerable to manipulation of the experience, censorship, or reluctance to express a wholesome and truthful experience.

Finally, this case study primarily relied on the interviews where the researcher is the primary means of data collection. While the positionality of the researcher has been disclosed, it does not protect the study against the researcher's biases in collection, analysis and reporting of the findings. Besides the biases of the researcher at various levels of design, data collection, interview and data analysis, another potential limitation is that the researcher is a graduate student who is interviewing department chairs. I, as a researcher, do not have hands-on experience in an academic leadership position, and I may not be able to fully understand as a person who has gone through the experience. Also, the power dynamic and position superiority of the department chairs may influence the quality of the interviews, and I may not be able to elicit the depth and breadth of the information that I desire.

\section{Summary}

In this chapter, I have explained the methodology and research design of this study. I presented my epistemological standing and explained why I have chosen the case study, Then I 
further elaborated on the data collection and analysis processes. Finally, I ended the chapter with a data analysis and trustworthiness discussion. In the next section, I will present the findings of this study. 


\section{CHAPTER 4: FINDINGS}

In this chapter, I report findings for the following research questions: "how do new department chairs experience transition from a faculty position to a leadership role?" and "how do new department chairs navigate the challenges of the transitioning to leadership?" In this embedded single-case study, I collected data from 7 chairs through two interviews with each and analyzed their resumes, calendars, and appointment letters. By binding the case study to the University of Missouri, the various data points helped me to delve into a rich dataset to capture the collective experience of the transitioning into leadership.

I utilized the Schlossberg's transition theory $(1981,1995,2011)$ as a guiding framework to cover the four pillars of self, situation, support, and strategy regarding how the faculty members experience the transition and navigate the challenges. I explored each participant's self and the situation in both the roles of a faculty member and of a department chair. Then, I investigated their perceived support systems and strategies while navigating the transition process's challenges. To better understand the self and situation of faculty members during the transition to chair position, I explored career motivations, tasks and responsibilities, and success metrics for both positions in company with departmental culture and professional interpersonal relationships. After defining the new chairs' career journey, I focused on each chair's election, nomination, and appointment procedures, as well as their formal transition and how they were making sense of the experience. After that analysis, I explored the new department chairs' challenges, and support system and strategies in navigating these challenges by probing into their preparation before and early after their formal appointment. The training and coping mechanism are partially self-driven strategies and partly provided by the institution and other support 
systems. The following are the salient themes of this journey that are emerged from collected data:

Theme I: Transition is a Multifaceted Departure.

- From faculty to chair: transformation of career motivations

- From scholar to manager

- Altered success metrics

- Multiple paths, one destination

Theme II: Leadership is Interpersonal.

- On the importance of departmental climate

- Transformation of power dynamics

- On the importance of interpersonal skills

- The greatest ally is found at home

Theme III: Chairs are Finding their Way.

- Service provided an initial window into academic leadership

- The predecessor's role in shaping the transition

- Learning the role through meetings

- Trial by fire: essential skills and lack of preparation

- Solving problems through alliances and consultations

In this chapter, I will provide a granular elaboration on these academic leadership transition themes. I will substantiate each theme with detailed evidence and direct quotes of participants. 


\section{Theme I: Transition is a Multifaceted Departure}

Transitioning from professorship to leadership is a substantial change; it is a metamorphosis of professional identity from various aspects. Professors departed from their original career motivations of teaching and research, and with that they departed from their day to day activities of flexible teaching and research hours. After transition, they were bound to office hours and responsibilities of a manager and a leader. With this departure, success metrics transformed. In this new role, chairs are no longer measured against publication, grants, and teaching outcomes, but are perceived successful if their departmental faculty and college dean are satisfied with their performance. Barbara shared:

I mean, my life has all transformed, my concerns, my work hours, even my social circles are different now. Yes, I still work for Mizzou, but everything is different now. I clock in at 8 every day and meet with tons of people with all sorts of problems, and I need to make everyone happy from the dean to my students.

In the following sections, I will substantiate this theme through three subthemes. These themes and subthemes are aligned to self and situation of Schlossberg's transition theory (1981, 1995, 2011). I will conclude departure with brief explanations of each participants' official transition procedures.

\section{From Faculty to Chair: Transformation of Career Motivations}

Different sets of career motivation inspired the participants to choose the faculty role versus the department chair role. Participants mapped the departure point after their graduation, and they shredded their carrier aspirations at the time. To a large extent, faculty member career choice was premediated and foreshadowed by proceeding events. Their main motivation to become a professor was to teach adults, advance knowledge, and enjoy the autonomous nature of 
a faculty life. Indeed, the passion to research drove participants to pursue their education to a Ph.D. level, while working as teaching and research assistants at university.

Embarking on an academic leadership path was motivated by completely different incentives. These professors felt the urge to step up and accept the role largely for altruistic reasons and moral-ethical responsibility. Essentially, they accepted the role because they either felt they were the most qualified or that they were the only eligible candidate in their department. Participants were also motivated by serving their department and having a greater impact on the student body. In the following section, I will expand their divert motivators.

What originally prompts an individual to pursue a career indicates their aspiration and expectations about a position. The participants reflected on the time before embarking on the faculty career and shared their aspirations for this job. Passion for teaching, desire to research, autonomy and flexibility of faculty responsibility, and expected career trajectory after attaining their Ph.D. among few others are the major issues that prompted them to pursue a career as a university professor.

The prevailing reason that prompted the participants to choose a faculty career was an innate desire to teach. The intentionality to pursue education is evident when the participants were asked to describe their motivations to choose this career. Genevieve reminisced on her teaching experience prior to her Ph.D. and explained how she someday planned to return to the classroom setting. Theodore described his desire to teach too when he talked about some professors from his undergraduate education; he wanted to make a difference in the world, and he believed he is able to improve upon some rather 
lackluster learning environments that he experienced as a student. Theodore continued with, "I love the research, but I do it to inform me to make me a better teacher."

Adam's family served as his motivation to pursue teaching. Nearly everyone in his family is a teacher; his parents and siblings consider teaching higher education to be very prestigious. He intentionally chose to work in academia over industry, knowing this path would enable him to teach. As a faculty member, Anthony was able to teach a subject that he was passionate about. He was drawn to this position because it allowed him to be compensated for enjoying one of his passions in a professional role. He found deep satisfaction in sowing the fruits of his labor seeing his former students succeed on various media platforms. He felt pride in his ability to shape student knowledge with his rigorous courses.

Research was the second most common motivating factor for individuals to pursue the faculty route. Margaret shared her initial desire to teach in undergraduate education and described how later in the course of pursuing her Ph.D., she discovered that research excited her and prompted her to further her career. Other chair member experiences paralleled those of Margaret, citing research as a provoking factor for climbing the hierarchy of academia. Genevieve believed that research was not a burden; rather she found it to be a creative outlet that influenced her practice and teaching.

Study participants desired more autonomy and flexibility in their careers and believed the advancement to a faculty position would afford them greater freedom and independence to complete their work. Barbara was exceptionally pleased with the flexibility of her role as a faculty member. She felt unimpeded by the rigid structure of schedule which she was accustomed to. Theodore echoed these same sentiments when describing the main source of his reluctance to move from a faculty position into an administrative one. Theodore owned a farm in 
another state, while simultaneously owning multiple consulting companies. Theodore mentioned, "I could do my research, and it didn't make any difference if I was in my house in Columbia, Missouri, on the weekend or at my house in Iowa. As long as I had internet access and the data, I could do my research.”

The above is what prompted these individuals to pursue a career as a professor; however, what prompted them to embark on the leadership in their departments were different. When asked about what prompted these faculty members to pursue a career in academic leadership, a handful of common themes emerged: moral duties and altruism, expected next step for a faculty member, desire to have a higher impact role, and personal exploration and growth are among them. Some believed this role for tenured faculty is inevitable and some seek financial incentives and taking a break from research through this position. Circumstances of the event including the timing of transition regarding department politics and personal life timing also facilitate this the transition of faculty to the chair role. In this following section, I will discuss these motives and aspirations.

During the interviews, it became evident that these professors felt their department needed them and they have to step into the academic leadership role. Some accepted the position because they believed there were no other qualified candidates for the job, while others genuinely thought they could salvage their department and make a difference in their campus. This moral duty to help their department in that situation prompted them to consider this position.

During her time as director of undergraduate studies, Barbara shared, "I was able to approach potentially divisive issues with the colleagues and get them to come together. I could be strategic about that and that I knew how to do it." She felt she should not 
withhold this from her colleagues in the department. For Barbara, not only did she want to contribute, she felt expected to do so. Many of her colleagues encouraged her to step into leadership by boosting her self-confidence to a point where she also believed that she could lead. When Barbara reflected on what persuaded her to become the chair of her department, she bluntly admitted that "it was clear there was really nobody else to do the job. I thought that I didn't think the department would be in good shape with somebody else as chair." She said, based on the department's qualification criteria, there were only a few candidates who qualified for the position. Her previous chair and senior colleagues were bolstering her belief that she ought to become the next chair. She confessed, "I was pretty resistant to that for a while," but later accepted it as a natural next step upon attaining her full professorship.

Likewise, Theodore expressed that there were no other feasible options. He recounted his colleagues approached him on various occasions saying, "we need a change; not just a change, we need you." It was hard for him to dismiss his colleagues' requests and selfishly focus on his teaching and research. Similarly, Genevieve also expressed, "I think part of it was recognizing that I had been really protected in my 8 to 9 years up to that point. I guess at some point, I think it's important to kind of give back to the department."

Many of the professors willingly stepped into the leadership role not only to have more tangible effects on student education but also to create a more positive impact on their colleagues' careers and their departments. After reaching tenure position, Margaret recounted wondering how she could contribute to the university beyond just concentrating on her own research and students. At that point in her career, she wanted to shift the culture and make a positive impact on her department. Marco talked about participating in some sessions with the 
Dean's office, describing how they ignited his passion for making an impact on his entire department and college. He grew curious about what he could do to make the university better.

Department chairs participating in this study confessed that they knew this job would be challenging. Theodore said he knew that it would be a struggle, but he kept thinking that "if I can make 43 of my former colleagues better and help some things happen, then that's having a bigger impact than myself being a faculty member."

After years of working in higher education, Theodore began to believe that he ought to put the institution ahead of all else. He believed ambitions directed toward improving his academic institution trumped his personal goals. Moreover, he expressed frustrations about other leaders operating under a self-serving agenda. He felt that it was his moral duty and a temporary commitment. He felt called to mentor his junior colleagues and pay forward the support he received from the department when he was an early-career faculty member.

Margaret's experience was similar to Barbara's; she also recently attained a career milestone, authoring a book, and afterward, it did not take much time to conclude that she would like to take on a leadership role rather than writing another book. She had a keen awareness that she needed to be chair of her department at some point, and the time seemed right to her.

Some considered self-realization to be the driving force to pursue leadership, while others considered the timing of these events a more critical factor in deciding to attempt attaining a leadership commitment. Margaret explained that due to the age of her children, pursuing a leadership role during this stage of parenthood was better now than delaying to a later time. She also talked about her own age and expressed doubt about her 
ability to succeed in this career path in 6 years. She said the timing for this commitment felt good. Theodore expressed similar life timeline issues. Earlier in his career, when he mentioned pursuing a leadership role to his wife, she asked him not to become a department chair. She worried that they just started to have children and believed the demands of this position would leave him unable to fulfill his family duties.

Some faculty members perceived this leadership position as a low-risk option to test the waters of academic leadership. Margaret said it was merely a 3-year investment, and if all went well, she might extend. She did not view it as a long-term commitment. Furthermore, it was not a burdensome position to attain, as there was only one other person competing with her. Adam had been in academia for more than 2 decades and only had a few years left. He had devoted his academic life to research and teaching, but now felt it should also include policy and decision making. Nearing the end of his career, he wanted to test out those areas with the option to decide later that leadership is not for him. He went on to say there are multiple ways that a leadership position may be vacated: they can leave voluntarily, be involuntarily removed, or quit the position, yet they are still able to return to a faculty member without losing out on anything. Overall, we have seen the difference between the motivation and aspirations in pursuing both positions and where these individuals stand regarding each position.

\section{From Scholar to Manager}

To understand the professional magnitude of the transition to the new role, I studied the day-to-day tasks and duties of these chairs and probed into their previous life as faculty. It became evident that the two roles varied greatly in tasks and responsibilities of their everyday obligations. When studying the routine tasks and common responsibilities of faculty members and chairs, the apparent differences were striking. Faculty workhours and location are relatively 
flexible, while chairs must be in the office at a certain time. Previously the participants were required teach and research, however these activities were minimized after transition, with most of their time consumed with administrative, managerial, and leadership duties. New chairs tended to spend time and effort on administration that their formal assignment required. In this process, either the research aspect of their career suffered, or they ended up working above regular work hours, which impacts their worklife balance. A fair portion of academic leadership tending to ad hoc issues, and chairs perceived "putting out the fire" as an expected part of their job. Consequently, chairs, who used to enjoy a great degree of autonomy and self-governance as faculty members, now have external loci of control regarding their careers. Chairs felt that in the current role, not only did they nurture students, but also their colleagues in the department. The following section will elaborate the differences in day-to-day life of a faculty members and a chair.

The faculty role generally allows a high level of workday flexibility and decisionmaking autonomy. They are able to structure their schedule based on personal preference and life demands. Research and course preparation can be done from home, on-campus office, or anywhere with an internet connection. Furthermore, work autonomy reached beyond mere day-to-day flexibility. Every few years, faculty members were allowed to take a yearlong research sabbatical and return to their position at its conclusion.

Theodore reflected on his time as a faculty member, describing how he was able to return home in the afternoons to take care of his children or check-in on his out-ofstate farm. His schedule allowed him to have extended weekends there, working from his farm. Genevieve had many different activities to balance as a faculty member: 
conducting research, publishing, presenting in conferences, collaborating with other researchers, and preparing materials for teaching courses. Genevieve's course load was typically two classes each semester, while other faculty members said their load ranged from 3-5 courses per academic year. With all of the responsibilities required by her job, the flexibility of the faculty position was essential to her success.

Aside from teaching and research, a large portion of time was spent on service projects. Service projects take many forms ranging from involvement in their own department and campus to local community service projects, all the way up to contributing to professional and academic committees at a national level. At the campus level of involvement, faculty would serve in roles such as on-campus faculty council and search committees. Faculty members also spent time reviewing journals and conference papers at the national level and participated in various academic and professional associations.

Typically, department chairs retain their tenured or non-tenured faculty seat during their appointment as chair. In this section, participants describe their life as chair and what compromised most of their tasks and duties. Understanding the present routines and work of chairs helps to better grasp the transition experience of faculty members into this role. All the chairs who were interviewed except one had almost half of the effort distribution allocated to administration and service, and about of a quarter of teaching and a quarter research distribution of effort. Theodore was the exception, who was appointed as $100 \%$ administrative chair. His positions had no requirements for teaching or research, while all the other chairs were able to retain some portion of their responsibilities as a faculty. Genevieve felt protective over aspects of her faculty position that she found most enjoyable. She could continue to conduct research, teach and keep her commitments to her service projects. These were the things that drew her to a 
career as a faculty member, and she wanted to keep doing them. Marco shared that he was assigned the typical work distribution of a faculty member but that this was only on paper. His day-to-day activities were consumed mostly by administrative tasks. Marco had just found out that some of his grant proposals were funded. He says he is able to continue his research, but it is hard to carve out time during the day to work on it. He has to prioritize it by waking up early and working on it between 5-7 AM each day. While serving as departmental chair, Barbara said she is still expected to have a research agenda, attend conferences, and publish, which exceeded the formal effort distribution.

The departmental chair workload is much different than that of a faculty member. Although the participants agreed to move into an administrative role, many of them felt protective of the job aspects that they previously enjoyed and attempted to prevent them from slipping away or becoming less important when faced with many new administrative tasks. Theodore mentioned that he was frustrated with the constant demands of his job, saying he is needed to address problems after hours and even on the weekends. On several occasions, he was out with his wife, and each time, he received calls regarding Title XI issues that needed to be addressed immediately. These kinds of burdens and responsibilities required Marco to cut down on his children's after school activities and some other family duties.

Barbara said as a chair she is constantly putting out fires. She enjoys this aspect of her role, calling it a fun part of the job. She said, "there are things that are unpredictable, and there is always some kind of new thing you have to figure out how to fix or how to solve or how to make things less bad." Unlike Barbara, Adam seemed indifferent about these duties as department chair. He said he simply accepted that putting out fires is an 
important job as a leader, many of which are personal problems of the people in his department and are the direct or indirect consequences of people's mistakes.

Margaret feels that she plays a role in improving people's lives and describes it as "my job is to create the structures that allow people to succeed and to dismantle structures that stand in the way of them succeeding." Margaret finds it extremely gratifying to see the fruits of her labor. As an example, she explained how she found an endowment that funded a portion of an undergraduate program in which she took pride. Like her colleagues, Margaret also appreciated the challenges of the role, which felt empowering when she realized that there were decisions left entirely up to her judgment. "When I make decisions that have positive effects on people, I think that's a very empowering moment, and that happens all the time in this position." For Marco, the best part of this job is the opportunity to advocate for his colleagues. He said that in his position, both his and the faculty member's success were intertwined, and that their failure directly reflected on him.

Compared with a faculty role, chairs felt they had lost their workday flexibility. Adam said, 'I'm here every day pretty much. You know, if you are a regular faculty, some days you may decide to come an hour late. Okay, we can't afford to do that." Barbara spends all weekdays from 8-5 in her office. In addition to losing the flexibility of work hours as a faculty member, the participants described feeling confined to their offices and university campus. Marco said, "I used to work from coffee shops all the time, so now I only go there to get coffee and come back."

Departmental chairs also have less flexibility and autonomy than they did as a faculty member. Those characteristics were essentially the most desirable features enjoyed by participants when they were the faculty. Barbara said, 
As a faculty member, really, I could have never come into work. No one would have noticed. No one ever knew what I was doing, you know? It was really just absolute autonomy, and I definitely don't feel that way now. Now, I feel like I am held accountable for my time and my activities way more than I ever was before. For Margaret, the main difference between being a faculty vs. a chair is that she now has much less time to herself. She said she pursued a profession in academia so she could read, write, think, and teach. Now the time she has to devote to those things is scarce. $25 \%$ of Margaret's workload is committed to research, and she continued with, " $25 \%$ of research appointment means one day a week work all day on your own things, and that kind of gave me a little bit of breathing from the chair, but things come up all the time." Genevieve carefully guards the $30 \%$ portion of time allotted to research and $20 \%$ for teaching. She ensures she is able to block time on her calendar for these tasks and leaves the rest of the calendar open for chair responsibilities. If one day happens to be heavily burdened with chair duties, she compensates for that lost time over the next few days with more research and teaching activities.

When asked about the differences between his previous role as a faculty and his leadership position now, Anthony shared that his top three concerns have shifted, "So before it was classes and grants and papers, now I've just traded those problems. So now, the three things that I deal with are money, space, and bad actors." For Marco, the biggest difference is that

As faculty, you're just worried about you, you and maybe your students and close colleagues. But here ... now, it's everybody's problems are your problems. Or you have to not just solve them when they happen, you have to predict if something is going to happen next month. 
Marco believes that now more than ever, his job is to advocate for all of his colleagues. He calls himself the cheerleader of the whole department. Despite the frustrations they experienced in the leadership roles, many participants found pleasure in their new responsibilities.

\section{Altered Success Metrics}

In order to understand the transition of a faculty member to a chair, success metrics and evaluation of these positions can shed light on the areas that faculties and chairs need to excel. Both faculty and chairs are formally evaluated on performance, but aside from these evaluations, there are other indicators they seek out to use for self-evaluation. By and large, faculty members are measured against two criteria: research and teaching quality. In addition to these benchmarks, interviewees revealed that awards, publications and grants, and respect from colleagues contribute to how faculty perceive their performance.

Chair formal evaluation differed and came in the form of an annual review. However, they seemed to value the opinion of their dean and acceptance by their colleagues as better markers of success. Interviews with chairs revealed that new chairs are happy when they can minimize a leadership fiasco in the department and leave a positive impression on their faculty. This is especially important because faculty members provide annual performance reviews of their chairs, and they vote to reelect their chair for the second term. I will explain in-depth these distinct success metrics for faculty and chairs in the following section.

There are many types of awards through campus and academic associations, which are nationally recognized indicators of accomplishment. Barbara's success at having her book accepted for publication was evidence that her research was going well. Similarly, Margaret felt a sense of respect in academia when her book submitted for publication was taken by a good press; she had a great sense of accomplishment. For Anthony, success was measured by papers 
being accepted without rejection. He felt his research productivity and caliber are high, demonstrated by increasing grant dollars; the influx of funds to the department was empowering and proved his capabilities.

Respect from colleagues helped some faculty members assess their effectiveness within their department, mentioning being approached for advice by colleagues who trust and rely on them as a key insight into their performance and productivity. Barbara explained, "people come to rely on me for a lot of things," and Theodore frequently has requests from both internal and external agencies asking his advice:

I mean relationships outside of the university. And the only way that I have those relationships is if I've been effective and can deliver with what I do inside the university, whether that's teaching, working on these grants, or projects that involve outside entities. And that's the way I know if people come to me with questions or projects and want answers. Then I feel like I'm being successful.

Theodore not only mentioned professional relationships that provide proof of his capabilities as a teacher, but he also went on to say that thank you notes from students reinforced his quality as a teacher. Anthony feels that his teaching is effective, evidenced by many students contacting him for mentoring and advice. He feels this is a great indicator of his success when students tell him how helpful his classes are. Participants shared that as a faculty member, annual reviews, awards, publications, and grants were used as markers for success.

Department leaders' success is measured differently from faculty members, and their perceptions about doing a good job looked differently in the new position. After interviewing the new chairs, it becomes evident that they place a lot of value on the 
impression their deans and faculty members have on their roles. In the following, I will explore how chairs perceive success in this role.

The new chairs value their dean's perception of their performance as paramount importance. Margaret meets with her dean and, together, they review feedback from faculty members regarding her leadership performance. Following this discussion, her dean then assesses her on different metrics, including student success, alumni outreach, and diversity initiatives. Margaret's performance is based on these metrics of success and also includes peerevaluations and self-assessment. Anthony's evaluations are similar, consisting of a departmentwide survey to collect feedback and Academic Analytics data (need to describe what Academic Analytics is), which his dean then discusses with Anthony to appraise his performance. The provost sets metrics, goals, and standards for Marco, and he is required to meet monthly with his dean to monitor performance and develop plans to successfully meet the objectives. Similar to other chairs, his goals include measures such as student retention, research expenditures, and semester enrollments.

Another important indicator that occupies the minds of the participants is how they are perceived by faculty in their department. They deem it as the ultimate indicator of their success or failure. Because she receives such positive feedback from her colleagues, Margaret feels satisfied with her job performance. She says, "the faculty really have been very supportive. Not all of them, obviously, not everyone comes in here and says, 'I think you're doing a great job' but enough to do that. I regularly feel like they reflect to me." Theodore takes pride in his leadership because he feels like people look up to him. "They set up meetings with me all the time. They trust me. They'll run ideas by me. I tried to be ... I consider myself an empathetic leader, and they appreciate that." Theodore continued, "If I give them the vision, they'll 
genuinely move in the direction that we want to go." In fact, Theodore gets invited to campus-level committees and meetings, which he interprets as members of higher-level leadership have faith in his ability to deliver effective and consistent results. In Barbara's opinion, the fact that she can bring more resources to the department and hire new faculty members at a time that budgets are tight is evidence of her success.

In sum, success metrics of chairs and faculty members are distinct from each other. While great research and teaching performance make faculty feel successful, chairs, to large part, perceive their success based upon the impression of their deans and colleagues in the department.

\section{Multiple Paths, One Destination}

Faculty members who participated in this study took different paths on their journey to becoming chair. Some anticipated this position, and other took the role quickly, without much notice. Some won competitive campaigns, while others were nominated by their colleagues and simply accepted the position. Still others were handpicked by their dean, previous chairs, and/or senior faculty to lead the department. Each faculty member took a vastly different path to becoming chair and none shared the same level of desire to hold the position. One chair applied externally for the position and went to lengths to move from his out-of-state university to take this job, while another individual was reluctant to accept the job after being persuaded by her dean, necessitating sessions of career therapy and consultation with family members before agreeing.

Competition and nomination were also different. One chair was handpicked by the powerful senior clique in his department, while another had to win an election among other candidates for this position. The expectation of the role was not universally 
expected to the same degree for each of the participants. One of the chairs knew she should take on the role the following year and shadowed her predecessor during the preceding year to prepare. This is in stark contrast for another chair who it took a few weeks from the time that he learned about the vacancy and officially started the job.

While some participants had almost a year to mentally prepare for their new role, others were at the opposite end of the timeline spectrum. For some, the process was extremely short, thrusting them into a leadership role in weeks to months. Marco recalled, "the whole thing from the time that you heard that you're the final person for the position to the starting day was only 2 weeks." All he had time to do was prepare a vision statement document, meet with the previous chair, and then it was time to officially start the role. When Theodore accepted the interim position, he had a few months from the time he was appointed until the start date. However, for his chair position, the dean sent out an announcement saying effective tomorrow, Theodore would be the permanent chair of the department.

At different points during the transition process, people perceived themselves as the chair. The signal that distinguished that their faculty role had concluded, and the role of the chair had begun, all took different forms. For Genevieve, it was signing the first fiscal email. Barbara only began to feel she was truly the chair once she saw the signed offer letter. She went on to say that after that moment, even some milestones, such as her first day in her office, felt anticlimactic in comparison. In contrast, that moment for Margaret came when she actually moved into the chair office in her department. Below chairs share their unique experience of competition and nomination for the position, and how they were making sense of the processes. 


\section{Genevieve}

Genevieve did not self-nominate for the position. Her colleagues put her name on the ballot. She was among some of her fellow colleagues with whom the dean met to see if they might be interested in pursuing the position, but none of them were willing. Left without an option for replacement, the dean called another department faculty meeting and proposed some less-than-favorable alternatives, such as hiring an external chair. When the faculty members expressed their dissatisfaction with this proposal, the dean chose to make the position more appealing by adding an assistant chair position to divide the onerous workload between two people. They called for new nominations, and from the handful of people who were nominated, Genevieve and another peer who ultimately became her assistant chair agreed to move forward.

Genevieve then met with the dean and associate deans for an interview, followed by a meeting with faculty for questions and answers. Genevieve was not expecting this type of transition into leadership. Her initial reluctance to take the position felt justified as she experienced the shock of the job. She felt flooded with future chaos and uncertainty she was expecting to happen. However, after the initial shock, she felt more excited and confident, thanks to transition meetings with the incumbent chair.

\section{Margaret}

Contrary to Genevieve's hesitance to take the position, Margaret fought hard for the nomination and was exceptionally pleased when she won the election among her colleagues. The process was structured and democratic. There was a primary vote in her department in which she and several peers were nominated for the position. Nominees were required to submit a campaign statement that was circulated to the entire faculty for 
review. Afterward, a forum was held where each nominee answered questions submitted by the faculty. Finally, an election was held. Margaret recalled the moment she learned the results:

I found out; I was in my office because they email the vote count to the department. And I saw the email, and then my friend texted me right away, and I saw her text. And I was in my office by myself upstairs. And I do remember thinking, Oh! I mean, I was excited! I was like. This is for real, you know.

Despite her excitement, Margaret felt apprehensive when she started the job, and uncomfortable with the authority that comes with the position. As time went on, however, she felt more confident with her jurisdiction and her capability to make decisions.

\section{Theodore}

In contrast to the democratic process in which Margaret was elected, Theodore's transition to department chair seemed more bureaucratic. Theodore was appointed to the interim position by his dean without any formal competition or nomination process within the department. A few powerful senior colleagues approached Theodore saying that the department needed a new leader and that Theodore was a qualified candidate. Theodore said, "And then they went to the dean and said, 'here's what we want to happen."' When Theodore accepted their offer, this group of powerful faculty members and the dean appointed him to the interim position. A year later, Theodore said this about the dean:

He came and met with all the faculty in the division and asked me to step out and said "I'm thinking about appointing Theodore. I wanted to hear your concerns." But there were no concerns. I had full support. And then he appointed me as fulltime division director. So, there was not an open search for the position. 


\section{Marco}

Marco seized the opportunity when he learned that the previous chair would be stepping down, and he would be running for the position unopposed. He said, "it was just me, and there was an empty box. So, I was like, ok, what's going to happen? You can't lose out to an empty box. That's pretty much it." Just a few days later, he heard back from the dean about his appointment to the chair position. Marco felt that his colleagues were unanimously supportive of him in this position. He felt trusted and confident that he could effectively lead his department despite the fact that there was only a couple of weeks until the start of the semester and his official start date.

\section{Adam}

Adam was an external applicant for the department chair position. Coming from another university, he had already applied for a few chair positions and prepared a comprehensive application package. His initial interviews were via telephone and followed by in-person interviews before he was offered the chair position at MU. From the time he applied until he was formally appointed as chair was approximately 7 months. Adam was anticipating this leadership role for a long time, but he did not expect the path to attain this position would be quite so taxing and arduous.

\section{Anthony}

When the chair position became vacant, Anthony used the Qualtrics portal to nominate two of his colleagues. The nomination process resulted in both Anthony and his two colleagues receiving the highest votes. This shortlist was pared down in a series of interviews with the dean and his associates, ending in Anthony's election. The process 
seemed nothing out of ordinary and provide enough time for him to ponder whether he really wanted to pursue the leadership path for the next few years of his career.

\section{Barbara}

Barbara was the only person in her department who was both qualified and wanted to be a chair. A committee was formed in her department that consisted of the chair and previous two chair members who were in the department to recruit the next successor. It was not difficult to narrow it down to her. Barbara's predecessor was inviting her frequently in her last year to important meetings, and her transition into leadership seemed natural and expected. Barbara felt well prepared when she started the job. She felt competent.

In sum, the experience of each faculty member in the process of nomination, competition, election, and appointment have been shared. Each person's experience was different from the others. This conversion from a full-time faculty member to a department chair, predecessors played a crucial role, and each had a unique style of engagement with the next chair. In the next, I will address how predecessors influenced their transitions.

\section{Theme II: Leadership is Interpersonal}

Relationships drive the faculty to take on the leadership role, challenge the new chairs, and empower the chairs during hardships. While well-liked faculty get nominated for the role, after sitting in the position, the new chair's relationship dynamics with the previous peer changes. After the transition, the chairs are required to make decisions for the department and evaluate the other faculty members who were previously their equal peer. Chairs are expected to inspire and unify the faculty, advocate for the department, and moderate the conflicts. Academic leadership heavily relies on interpersonal skills and a chair must connect to and communicate with different constituencies in and outside their home departments. Margaret said: 
This job is all about people. You know, they should like you, otherwise you won't even get elected. You should like people too and maintain good relationships to be able to work with them. At the end of the day, you are managing people.

In understanding the transition to leadership, chairs' relationship with their family also impacted the transition to leadership. This theme touches on all the four facets self, situation, strategy and support of Schlossberg theory of transition (1981 \& 2011). Below, I will elaborate the various relationship components of transition.

\section{On the Importance of Departmental Climate}

The participants discussed their hesitation with taking a leadership position during periods of uncertain intradepartmental dynamics. Historically, they might have experienced conflicting values and disruptive teammates who hindered collaboration and collective morale. However, before assuming their new title, chairs found their work environment generally healthy, with colleagues and other leaders mostly supportive. New chairs also perceived the current culture and political dynamics of their department and school healthy and positive. All new chairs expressed that the faculty members in their department are supportive and collaborative. If not so, they would not have accepted the role. Some delayed consideration of leading the department and waited for culture shifts in their favor. A few explicitly mentioned that they would not accept the position until their "toxic colleagues" and "bad dean" retire or leave. Despite recalling some historical adversities, new chairs perceived their department as healthy during their term of service and the culture as collegial and supportive.

When asked to recall their mindset prior to accepting the offer to be chair, each chair believed they were in a healthy state of mind and felt supported in their decision. 
Some mentioned that the very reason they considered the role was entirely due to the positive encouragement and supportive climate expressed by their colleagues. Genevieve acknowledges that politics are everywhere; despite that fact, she believes her department is pretty healthy. To her, a healthy department is one where everybody talks with each other, lines of communication are open among faculty members, and differences in opinion are represented equally. She goes on to mention that there are several groups of people in the department that socialize outside of the workplace, which she takes as a good sign. Another indication that the department culture has a positive impact is the department's campus-wide performance evaluation, indicating that faculty members are productive and generative. The current workplace culture that she strives to maintain is the product of her experiences as a junior tenure-track faculty member. She said she felt "protected, sheltered even." The department encourages new members to learn and grow. They were allowed to write and establish research agendas without being bogged down with burdensome programmatic tasks.

Margaret also believes she has a healthy work environment, describing it as being an intellectually stimulating community filled with smart, vibrant people who graduated from prestigious universities. According to Margaret, most faculty members take their research very seriously, many having won awards. Multiple faculty members in her department also serve as leaders in the campus community. As critics by trade, they create healthy friction within the department, ultimately resulting in improvement.

Theodore called the climate of his workplace collegial and went on to say it had been more than a decade since anyone yelled in a faculty meeting or got really angry. It feels there is a healthy tension in the department that pushes people to grow together for the betterment of all. Outside of a few individuals, Adam thinks the faculty members in his department are collegial 
and largely supportive of one another. Marco has not had any concerns about the department's culture. He describes his working environment as positive, adding the ease in which he is able to collaborate on almost all of his grants and research with his colleagues in the department. If he receives proposal rejections or any other setbacks, his colleagues are very supportive if he needs someone to talk to.

Barbara and Adam both agreed that as faculty members, they used to meet and talk to their colleagues on a day-to-day basis for team building, collaboration on research projects, department issues, and even social events. Genevieve and Marco had similar experiences in their larger departments, saying their life as faculty members did not feel isolated, adding that they interacted with their peers far beyond the monthly faculty meetings.

Historically, it was not always a healthy culture in their departments and the chairs continue to struggle with some of these difficulties. Barbara does not believe that chairs have always been representative of all faculty members, which alone led to bitterness and resentment among the group. Cliques formed within her department, and everyone suffered from the divided culture. Her goal as chair was to be a chair to all, not just some.

Conflict arose in Anthony's department when his previous dean and senior faculty members resisted change because they preferred to continue traditional, outdated protocols that no longer fit the department or its faculty. Barbara, Theodore, and Anthony all mentioned that the culture in their departments has improved since the departure of some difficult faculty members, some retiring and others leaving for other schools. Barbara said: 
It's changed a lot. It's gotten better. We're not alone in this. I mean, we had a couple of extremely toxic colleagues who bullied other faculty members and who made it really is, kind of made it, difficult for the department to get together and do things as a group.

She mentioned that she could not be a chair over those people if they were still there. She continued with, "People didn't want to be around them. Or they would come, and they would be hostile and demeaning to other colleagues." Reflecting on culture previously, Theodore remembers, "the culture was very adversarial. I still remember the first few faculty meetings when I went to with screaming matches and yelling matches in there between faculty members." Theodore also had that misalignment and only considered this leadership position once those people retired.

Overall, department culture is supportive and collegial at the time the faculty members were considering the position. This positive attitude of the chair toward their department is not accidental. These chairs mentioned that they would not accept the position if their departments' climate was not what it is now. Some waited for the proper time for some colleagues to leave or retire, and some waited for a change in leadership to take on this journey when the leadership above them are in line with their visions. To better understand the ecosystem that chairs are functioning in, I will also explore the professional relationships of the chairs before and after accepting the position in the next section.

\section{Transformation of Power Dynamics}

The relationship of the new chairs and how they perceive new role has affected their existing relationships with others, and the new relationships are important to understanding the transition of chairs. New chairs perceive their relationship with department faculty members have changed because now they supervise their pervious peers. Needless to say, chairs also make 
important decisions for the faculty such as promotion and tenure evaluation. Faculty members feel a shift in power dynamics and subsequently interact with their chair differently. Now chairs have access to information about people they did not have before and cannot share openly all matters with everyone Also now, chairs are perceived by the faculty as the voice of the administration and the individual who implements university policies at the department. One chair shared that her department believes she has moved to the "dark side," the administration.

While old relationships have changed for some chairs, nearly all participants described newfound connections on campus as a result of rising in the campus leadership hierarchy. Barbara was very pleased with finding a new community among other chairs on campus. Genevieve marveled at her blossoming resource network of leaders across campus to whom she would never have had exposure before. Marco's professional circle grew, adding interactions with distinguished alumni of his department, collaborating with members of the advancement office, and working closely with human resources staff. Chair members were pleased to witness their professional networks flourish in their new role.

Chairs experienced shifts in their relationships with their colleagues in their department. They are required to supervise, evaluate and make decisions for people who were previously their peers. Many participants felt that after the transition, their faculty members do not look at them in the same way. This change in social life seemed traumatic for some. Power dynamic between incumbent chair and faculty members has changed. 
Sometimes appointment to leadership creates distance in collegial friendships. Anthony accepted the job under the assumption that he would have fewer friends during the course of his leadership. He feared that he would make decisions that would not be well-received by all and that he may do things that could make some people unhappy. Anthony was not alone in his struggle with making difficult decisions concerning longtime colleagues and friends. Margaret, Genevieve, and Marco also grapple with it. A few of Genevieve's in the department approached her, saying, "I know that you're the chair, I'm not going to try to take advantage of our friendship." In her few short months as chair, Genevieve had been required to make difficult decisions that may impact the inter-departmental relationships. And initially, Margaret felt that her friendships in the department became more distant and that they withdrew from her. However, as time passes, she is growing more comfortable with this shift. Genevieve shared that: it can be a very isolating job because you're a faculty member, but you're not in some ways because you have these decisions to make about annual review and workload and other things. So, while you are a colleague, you have these other responsibilities that kind of create a power dynamic as well. You are often aware of issues or HR [human resource] things in the department that you can't talk about with others.

With the newfound leadership responsibilities and changing power dynamics within the department and the nature of this role, the participants felt that they needed to withhold more information, which they would have previously discussed freely with their colleagues. They lacked the transparency in conversation that they once had in order to prioritize the privacy of other members in the department. Human resource matters are considered confidential, and the information to which chairs are privy is legally prohibited from being shared. Genevieve sharedthat: 
things are going on, but you can't talk about it with your colleagues. So, there will be times when a friend and I would go to lunch, like earlier this week. I can't share what is in my mind, like bothering me. I'm working through something right now, and I'm having to deal with it, but I cannot have a more transparent conversation. Margaret describes a healthy relationship with her best friend in the department and believes she is a very trustworthy person. Nevertheless, Margaret feels they can no longer discuss work, especially information about other faculty members, even just in passing. She is cautious in her conversations, ensuring she does not inadvertently share sensitive information regarding their mutual colleagues.

Adam came from another campus when he accepted the chair position at MU. Unsurprisingly, the effect of taking the position of existing relationships was not a concern for him, and not all chairs felt that they were jeopardizing their friendships and relationships with their peers. Anthony said his relationships with peers had not been affected by his changing job role. Rather his time available for socializing was limited due to the time demands of his new leadership role. Few others also could not relate to the fears shared by some of their colleagues as neither noticed a change in social dynamics nor felt that their existing relationships had been affected by their change in roles.

\section{On the Importance of Interpersonal Skills}

“They're all interpersonal," Margaret said when asked about how to be a successful chair. Chairs must possess strong people skills. New chairs believed that their success or failure in this role it is contingent on how well they can relate to people. As leaders, they should be able to understand people, empathize with them, and create 
effective interpersonal relationships. Chairs should have the ability to work well with people. As a chair, Barbara said, "you have to have people skills. This is a weird term, but you have to have some ability to talk to people, listen to them, and work with them." Adam mirrored those sentiments saying that if a chair cannot understand the motivations of stakeholders and communicate effectively with them, that chair will be ineffective.

Participants went on to emphasize empathy and the importance of taking time to understand the problems and challenges of different stakeholders, including campus administration, faculty, students, and alumni. Essentially, they perceive their role as chair as a support person for the goals of the department. For Margaret expressed that it goes without saying, when a person is in the chair position, the person must be nurturing, attentive, and able to bring everyone together to work toward the common mission.

Chairs view themselves as moderators of intradepartmental conflict, some even feeling challenged by addressing issues in this area. They feel as though they are personally responsible for healing the historical fractures between departments and building a new culture that reduces further tensions. Adam described his department as having a division with groups comprised of people from two opposing disciplines. He went on to say that since the day he was appointed, it has been a struggle to bring harmony between the two groups. There is a disjunction between his faculty members who tend to research individually, making him constantly ask himself, "'How do I convince them to open up to collaboration? Because this is part of our success!”

The participants have chosen to promote full disclosure on issues of importance to their faculty and stakeholders. Some routinely updated their faculty and shared vital information with their advisory board to promote intradepartmental conversation. Theodore uses bi-weekly briefings sent to his department faculty to keep all parties informed, while Anthony's strategy is 
to regularly present updates to his advisory board. On transparency, Marco said: "it is better to be upfront. We are transparent on the budget and share everything with faculty so that there are no secrets." Genevieve intentionally creates an open space for people to share their concerns during faculty meetings. She wants to minimize unspoken issues, causing anger to fester and frustrations to be vented in less desirable ways. Marco takes a similar approach, making himself available to students and faculty to discuss their concerns in-person.

In recognizing that they alone are not responsible for the fate of the department, they are able to recruit participation of the faculty, allowing them to take ownership and pride in tasks that benefit the department as a whole. "I delegate well good to the right people at the right time to get things done," Theodore mentioned that. He continued by saying that task delegation effectively helped him to be able to deliver quality work and accomplish more than he would be able to do alone. Barbara said: "I've really enjoyed figuring out what everyone's good at and where they can contribute." She utilizes her delegation skills to get people invested in departmental matters. Chairs found that to build relationships they should be vulnerable and transparent, and with that they are able to build consensus among faculty members and boost collective morale in the department.

Prior to their appointment, some chairs sensed that their department was divided. After their election, they felt it was their duty to unite the department. As a foundation for proper functioning between colleagues, they made the unification process a top priority. Barbara recalls deliberately investing a great deal of her time and energy into bringing people together and reshaping the department, fostering the idea of one united intellectual community, and eradicating the divisions and cliques in her department. After a year of 
purposeful work toward this process, she believes she was successful in achieving that. Marco believes one of his greatest strengths is team building and the creation of synergism. In fact, he enjoys persuading people to his point of view as a leader. Margaret's department contains many programs with their directors and heads. She follows collaborative leadership models and creates committees made up of disparate groups of people in an attempt to bring unity and collaboration to the large department. She wants the different programs to feel unified with one another in working toward a common goal.

Some new chairs noticed faculty members who felt marginalized from the larger group and others who seemed disengaged from their research. Barbara considered herself skilled at elevating the morale in her department by identifying and reaching out to the people who were uninspired. Barbara felt she positively influenced the work morale of her department using this method.

When Margaret was appointed to the position, she felt that the faculty members were shaken by the major budget cuts and lay-offs of a significant number of their non-tenure track faculty member colleagues. In her opinion, this dispirited climate affected not only the faculty, but graduate students were also experiencing low morale. For the first year, Margaret focused on restoring the former energy of the department. She frequently met with the faculty one-on-one, asking how she could assist each person and the department overall. She says she is always brainstorming on ways she can act in her leadership role to improve the lives of people in her department, believing she should use her interpersonal skills to boost morale among her faculty and staff. Overall, chairs believed the interpersonal qualities of communication and unifying and boosting the morale of people are the must-have qualities for a leader. 


\section{The Greatest Ally is Found at Home}

Outside of their support system within academia, spousal support ranked highly among the participants as one of the greatest assets during this challenging transition. They acknowledged their spouses in supporting roles ranging from career resources, consultant and confidant, to emotional support and assumption of greater domestic responsibilities. The chairs discussed with their partners how their family will experience the change with the expectation of a greater level of professional responsibilities should they accept the role. Should spouses be unwilling to support faculty member's pursuit of leadership, these chairs may have not accepted it. After the initial green light from family, chairs shared that their family was their safe haven. Experiencing the toil and turbulences of the transition, their families are their all-time ally that without their support, they would not survive.

Recognizing the discord between the time commitment of the job and his family responsibilities, Theodore sought his wife's counsel often both before and after his appointment. He wanted to make sure his family was on board for this transition, noting that just a few years prior, he would not have been able to consider this position due to the young age of his children. Similarly, Marco acknowledged the sacrifices his wife had made and the toll leading a department had taken on their family. Genevieve shared that It is very emotionally taxing. I would say my husband was probably the largest support during this time. He listens every night and lets me just get everything out. He's also been understanding if I need to answer emails or to talk to someone from work on the phone at 8 PM., for example. Which has been typical for me as a faculty member. 
Margaret shared that her spouse supported her by assisting with problem-solving and became an audience for discussing more sensitive issues. He helped her with time management during chaotic periods and the unraveling of complex tasks. She described her husband as emotional support to help her feel calm, more relaxed, less stressed, and overwhelmed by the obligations of her position. Almost all the chairs acknowledged the significance of emotional support during their transition to leadership role.

\section{Theme III: Chairs are Finding their Way}

The initial exposure of faculty to leadership at higher educational institutions was through serving on councils, taskforces, and committees. Service also helped the faculty members expand their professional network, which later became instrumental in their survival as chair. In addition to pre-exposure opportunities through service, predecessors had a substantial impact on how new chairs experienced the transition. An incumbent chair can provide a multitude of training opportunities and pave the way for the incoming chair. As chairs assumed their new role, most felt insufficiently prepared. In the early months of the job, they had to climb a steep learning curve in some areas such as higher education finance and human resources. Chairs gradually learned leadership through meetings with other leaders and consultation with non-academic personnel. Chairs developed strategies that ally with leadership on controversial and challenging issues, helping them navigate the transition period better when frequently consulting with subject experts. Adam said:

I noticed that there is a huge learning curve at the beginning. The previous chair hugely helps me a lot to figure out what is going on. Nowadays, I am attending a lot of meetings... and whenever I don't know I don't hesitate to reach out to our staff. 
This theme reflects the Schlossberg $(1981,2011)$ 's coping factors of strategies and support. Chairs based on their self and situation utilize some common strategies and they seek or receive particulars support. In the following sections, I will specifically reflect how chairs are navigating this space.

\section{Service Provided an Initial Window into Academic Leadership}

Beyond research and teaching, the faculty are responsible for contributing through service and administration. The participants of this study were involved in leadership and membership of different councils and taskforces on campus before accepting the job as chair. Through service, they were exposed to academic leadership and administration in higher education settings, but also were able to meet various groups beyond their peers in the department and beyond. They capitalized on their familiarity with different entities and individuals on campus in departmental leadership and were aware of the university's mission and developed interdepartmental connections to they could reach out if they encountered questions or problems.

Their service, which accounted for approximately one-fifth of their responsibilities, gave the faculty exposure to policy and mechanism in higher education, a taste of leadership at different levels, and primed them to take on their next career leadership role. As faculty, they were engaged in service as committee members and leadership positions on these councils, associations, and committees. Barbara served for a year as the chair of the campus writing board. Both she and Margaret were directors of undergraduate studies in different departments. They also chaired other committees, including the tenure and promotion committees. Genevieve became the director of a prestigious certificate in her college and chaired multiple task forces and committees. 
Adam chaired a faculty search committee and served as associate head of academic affairs.

Theodore was the director in one sector of MU with broad outreach potential. Anthony served as president of a national association in his field with more than 2,000 members and led an enormous lab filled with high-tech machines. In essence, the service portion of the job provided some leadership opportunities that helped faculty to somewhat prepare themselves for the next role.

With more than 2 decades in academia as a faculty member, Adam has had tremendous exposure to both positive and negative examples of success that have shaped his understanding and vision of leadership. In duties beyond his faculty role, he was involved in administration. His meetings and project collaboration with his chair endowed him with lessons in leadership. At the university, he participated in a leadership curriculum through the university's provost's office, which consisted of ongoing training and meetings that he attended over a 2-year period. As an external hire, he was assigned to the program, but he also elected to attend a retreat for academic leaders to further hone his skills. Adam found these programs to be eye-opening experiences in academic leadership that better equipped him to have more meaningful discussions with deans and the provost concerning higher-level administrative matters.

Much like Adam, Marco sought out leadership training prior to his pursuit of academic leadership. During his service, Marco had the opportunity to see the intricacies of higher education administration. It was important for him to understand budget and policy issues, so he attended annual meetings to increase his knowledge on these topics. He also attends a national program hosted by his professional association, which aided him in the pursuit of academic leadership. 


\section{The Predecessor's Role in Shaping the Transition}

Predecessor influence on the transition of new chairs stood out from all other types of supports that new chairs have received. Predecessors have the potential to offer hands-on experience through shadowing opportunities, mentorship, and coaching. Many of the participants' predecessors played a valuable role both prior to and during the initial transition into their new role. This support, though, varied widely. For some, the predecessor provided an intentional succession strategy for them, while others had an abrupt transfer of power with little support during their transition period. It ranged from a predecessor who provided a year-long, full-fledged succession plan with plenty of opportunities for shadowing and shared decision making, to a predecessor who held one meeting and exchanged a few emails before a clean hand-over. New chairs must also inherit the projects and challenges predecessors leave behind from policy to various managerial and financial matters.

One area the participants recommend new chairs utilize is shadowing other chairs before accepting or starting an official appointment. It was the expectation that Barbara would become chair when the incumbent chair's term was complete. With that knowledge, she began shadowing him for the better part of a year, and as time progressed, she accompanied him to more meetings. While not completely certain about her appointment, this shadowing solidified her future position and made the transition easier. Barbara was groomed to become the next chair. Her predecessor wanted Barbara to be the next chair and prepared her well, so her transition happened more easily than most. During the last year of his leadership, she accompanied him to many important meetings, such as annual budget meetings with the dean and monthly council of chairs 
meetings. He included her in departmental decision-making and explained his rationale in detail. At times, he even sent her as his substitute. Her presence in the meetings was so great that on some issues, it may have even exceeded the presence of the incumbent chair. For that year, her predecessor asked for her input on decisions, actively integrating her in important meetings. All these happened, despite knowing there was no guarantee that Barbara would become the next chair. Now, as chair, Barbara still feels supported and mentored in her role. To her, it is an institutional resource, giving her the ability to look back on historical decisions to help resolve issues within the department in the present. She frequently approaches her predecessor and other past chairs from her department for any questions or coaching she may need.

Similarly, Theodore also experienced a preparation period where the current chair primed him to become her successor. He said she shared a lot of information that would only have made sense if he were poised to become her replacement. His predecessor shared difficulties within the department, explained the varying angles, and asked for his input on decisions. She also disclosed to him many of the problems that she was having with various groups of people in the department. When Theodore took over the position, he felt well-prepared and was confident he would be able to effectively navigate the political dynamics within the department.

In the months leading up to her appointment as chair, Genevieve regularly met with the current chair. These meetings grew in length and frequency, culminating in her role as department chair. She strategized her transition to leadership by compiling a list of topics and questions to ask for guidance regarding important matters vital to the success of her department. Together they explored issues regarding the academic calendar, major deadlines, organizational policies, institutional rules of the campus, and system-level mission and strategy. 
Not all participants experienced such a smooth transition into the world of leadership. Unlike Theodore and Barbara, the remaining chairs' predecessors did not have candidates in mind for a successor. Higher levels of uncertainty surrounded the appointment and election processes for the other participants and were therefore unable to prepare them in the same ways. Although they were not groomed to be the next chair of their departments, both Anthony and Marco received guidance while assuming their new roles. After their appointments to the chair position, they both described being frequently in contact with the incumbent chair via email before and after their start dates for clarification of responsibilities within the department. Each held meetings with their predecessors to discuss unfinished projects, current status of various departmental issues, and the vision for future advancement. Another chair took a more innovative approach, forming an advisory committee of previous chairs who, to this day, continue to aid him in his leadership.

Overall, the participants' relationship with their chair and departmental expectations of the next chairs and decision of the incumbent chair to provide support appeared crucial in how new chairs experience transition. Some predecessors wanted to offer more assistance to create a smoother transition for the coming chair, but the latency of the election did not allow them sufficient time to prepare the next one. Predecessors' preparation for the leadership role is not the only type of preparation for departmental leadership. Beyond the created opportunities by predecessors, in the next section, I will explore how new chairs had prepared themselves to be a leader in their departments. 


\section{Trial by Fire: Lack of Preparation}

Despite some preparation beforehand, overall, it was limited. There was a general consensus among chairs that more training was needed before the appointment. By and large, chairs walked into role unschooled in several skill areas such as, higher education finance, human resources, and advancement. Even when a newcomer assumed that they were competent in an area, for example because they excel in budgeting and personal finance, they had quickly learned that higher education finance is wildly different and very complex. Chairs were collectively baffled to learn about the various accounts at the university level and different restrictions access to each one. They encountered challenges in human resources when they were unfamiliar with HR policies and procedures on campus. Prior to this role, nearly none of them had been involved with fundraising and advancement. They felt uncomfortable and ill-equipped to be involved in those engagements.

Margaret said: “There's a huge learning curve. There's just a ton of new stuff to learn.” Barbara said she was not knowledgeable about many of the duties for her job as a chair until she started her appointment. Margaret also said:

I don't think there was anything on the campus that prepared me for it. I've had a lot of support since I became chair, but there was not a whole lot built-in to teach me how to be a chair before that.

Theodore expressed his astonishment with the lack of onboarding for administrators and leaders, and Genevieve shared that "there was no specific training, not before I was named chair." The collective expression from all participants was that there is a steep learning curve that the new incumbent chair must conquer specially in finance, budgeting, and human resource practices and policies. Theodore expressed his frustration with "finance has taken me a while just 
because of the flow of money around here and HR. There is no rhyme or reason in HR. I still can't figure out HR." These feelings are illustrated when the participants spoke of tackling the learning curve at the beginning of their appointments. There are also some idiosyncratic challenges for each chair and situation that are reported in this section. Below, I expand on these common and idiosyncratic challenges.

Faculty members usually do not have frequent interactions with alumni and alumnae and are not expected to conduct fundraising campaigns. However, some outreach and advancement are expected. The participants shared that they feel incompetent, inexperienced, and untrained in this area. Margaret admitted she had not mastered fundraising and had not done any outreach except for the bi-annual meeting with some alumni, while Theodore said the ins-and-outs of advancement baffle him. Barbara and Anthony also did not initiate any effort in that regard except attending one or two meetings with alumni. Anthony shared: “we've had at least two predecessors in this role who were masters at it. It seemed people just fell out of the woodwork, and not only did we have a donor, but they're giving us $\$ 8$ million!”

Financial understanding of the department and college budgets appeared to be one of the most important, albeit one of the most challenging, areas for the new incumbent chairs. Margaret learned that her dean believed successful chairs know the outcome of every dollar spent in their department by the end of their first year. She described it in this way:

It's really important to know where the money is because you can't understand how the larger machine works unless you understand the money that's driving it. If you don't 
know how the money works, then chairs are just constantly getting information that they can't understand the repercussions of.

Collectively, chairs who participated in this study expressed their shock and confusion at the complexity and multitude of accounts, distributions, and allocations of funds across campus. Before accepting the position, Anthony thought that he had an excellent understanding of money and the school's financial structure but went on to say:

I just found out yesterday that the school alone has 812 accounts. My first response was, 'Wow. For what? What do all these 812 accounts do?' And from some, you can spend freely, and there are some restrictions on others." Theodore said: "I had no idea of the scope of the finances of a division like this."

Adding to the complexity of account restrictions on various funds, new budget models and budget cuts have exacerbated the challenge for the chairs. It is necessary for chairs to understand the current budget practices in which their departments are operating, then learn and implement the changes in a new budget model. With the institution-wide budget cuts, the expectation was to shrink expenditures to apply the funds strategically within new budget constraints. Barbara admitted to having no formal training in accounting, finance, or spreadsheet management but was expected to perform in an area in which she was unfamiliar with the terminology and procedures. Her higher education financial challenges elevated to a whole new level when her fiscal officer was out for a few months and was unable to be replaced. She felt lost, and until some staff from the dean's office stepped up and resolved her questions and issues.

The participants did experience other unique challenges and skill and knowledge deficiency that are less commonly mentioned by others. These include persuasive and diplomatic 
speaking techniques, human resource compliance, institutional policies, and resolution of non-academic staff conflicts.

Anthony did not hesitate to say: "space, money and bad behaviors" were his greatest challenges after assuming the role. Theodore went on to illustrate these sentiments saying he was warned of the issues he may encounter with space at the beginning of his role. In the last few years, it has been necessary for Theodore's faculty and staff to shuffle their workspaces to accommodate another program that has merged with their department and moved into their building. A significant amount of his time and energy are spent convincing people to relocate to different spaces and resolving their issues and complaints. He further explained that faculty dissatisfaction with office space affects their relationships with Theodore as well as the dynamics within the department. Marco mentioned similar difficulties with space issues resulting from budget cuts. His department required a reduction in space and needed to justify the efficient use of the remaining space. There is a space usage committee in place; however, leading decisionmaking processes and pitching ideas bring all faculty to agreement is one of the most significant challenge areas at the beginning of his position.

The external chair who participated in this study shared that he struggles with evaluating his faculty, specifically tenure and promotion since he is not familiar with the faculty members' work. He is learning those tasks through institutional and college rules and policies, but he feels there is a large learning curve in this area for him. At the beginning of her appointment, Barbara had several colleagues who were eligible for promotion or tenure. There are many policies and procedures involved in the evaluation for this type of promotion, and it was essential for her to become proficient in 
assessments and recommendations for tenure. She sought out answers from many different people and reviewed extensive lists of documents to learn the process on campus. The challenge was so great that more than one of her colleagues recognized her difficulties and described her as being "baptized by fire."

Human resource issues presented a challenge for one chair who felt no one prepared him for this aspect of his career. He shared experience regarding faculty related HR, saying: "when it comes to staff exempt and non-exempt staff, I'm still scratching my head on that one." One chair vulnerably confessed that he is not an articulate speaker. He grew up with vernacular and speech habits that would be deemed politically inappropriate. He is conscientious of what he says and is careful to prevent them from emerging in his speech in ways that might lead others to draw undesirable conclusions about him.

In sum, chairs felt ill-equipped when it comes to fundraising and advancement engagement. They had minimal experience reaching out to alumni and donors for the purpose of donating funds to the university in their previous roles. Budgeting seemed a challenge for most; however, it shocked the ones who thought they have accounting and finance knowledge. Higher education finance was a different type of beast for most, and complexities of different accounts and types of access baffled them. Space management stood up as one of the main challenges for most of the chairs, which was intertwined with politics and persuasion power to make people move. Beyond these common challenges, each chair has their own particular difficulties in leadership. As mentioned as examples above, one was struggling with human resources compliance; another was grappling with managing their staff. 


\section{Learning the Role Through Meetings}

Chairs shared that majority of their learning has happened after accepting the position and starting the job, through meetings with deans, associate deans, other leaders, and various staff in the college and institution. The meetings at college level with other leaders provides meaningful exposure and networking opportunity. Chairs'staff and non-faculty colleagues in the department were vital to the chairs' success because they carry years of institutional knowledge and practical expertise on administrative duties.

Deans and associate deans proved to be great resources for new chairs to learn about expectations, desired practices, and acclimating to the new conditions. Some colleges have built-in programs and meetings specifically to train new chairs within the college. Barbara and Margaret both attended bi-monthly meetings for new chairs. Their dean met with them to address knowledge schemas important to their success and invited expert guest speakers to present on topics in those areas. These formal, college-level meetings included presentations on subjects such as budget, academic analytics, faculty and staff evaluations, and advancement. To supplement these formal meetings, new chairs attend informal lunches with associate deans to discuss leadership goals and approaches to navigating challenging situations that they may have encountered. These informal meetings provided the chairs with training resource support from the dean's office, as well as networking and collaborative opportunities among new chairs within the college. Margaret and Barbara both viewed their monthly chair council meetings, combined with their exposure to other chairs in the college, to be a tremendous resource for learning and understanding leadership in academia. In contrast to Margaret and 
Barbara's experience, other chairs in this study did not report required participation in meetings dedicated to new chairs.

The department Anthony chaired had no structured training programs or meetings that he was required to attend; however, he did have regular meetings with his dean and networking opportunities with the chairs in his college. His monthly meetings with the dean were less official. They met one-on-one to discuss expectations and concerns, and to develop strategies to overcome roadblocks he may have encountered. Anthony also made time to meet monthly for coffee with other chairs in his college. The combination of his alliance with the dean and fraternization with colleagues was useful to get him "up to speed" at the beginning of his leadership role.

Theodore and Genevieve participated in monthly meetings in which all the chairs in the college, the dean, associate dean, and fiscal officer were present. While the meetings were not solely focused on leadership, there was an opportunity to discover other leaders' thought processes and become familiar with their philosophy on leadership. Anthony emphasizes attending provost and department chair meetings expands this capacity, enabling an individual to rely on them if a future need presents itself. Genevieve and Theodore also have monthly one-onone with their deans who are great resources for questions they may have and to help troubleshoot their issues.

The information sources the interviewees found to be vitally important were their administrative staff. As one of their pivotal learning resources for their role as chair, Margaret said, "I have an assistant who's also the fiscal officer. That first year, I drove her crazy. I asked her everything, you know because she had helped him [previous chair] for six years, so she knew a lot." Theodore shared that when he started the job, his saving grace was his great staff, who 
helped him navigate the system and figure out policies. Some staff members had a long history in the institution and could provide context for many past decisions and policy creation. Genevieve's staff helped flag issues with documents to bring to her attention. Their support and investment in seeing the department succeed during the early days in the chair position helped build her confidence and elucidate some of the finer details required by her new role. Overall, staff had years of administrative experience and accounted for great resources to teach and support their chairs.

\section{Solving Problems through Alliances and Consultations}

In general, problems and challenges are inevitable in a leadership role, more so for the new leaders. Interviews with the new chairs have revealed that chairs create alliances with leadership and consultation experts in related domains as their two main strategies for overcoming challenges. To navigate the challenges and turmoil of the position, participants were quick to learn it was necessary to create alliances with the upper leadership to support their difficult decisions. To do so, chairs would share their plan with their leaders, and after securing their buy-in, they would execute the discussed set of actions.

During times of challenge and difficulty, alliances with leadership was one of the main strategies that chairs found useful. They meet with other leaders to seek agreement with their decisions before executing them. With the support they secured through this alliance, they were able to confidently navigate problematic situations. When Theodore was in his first year, he was hesitant to become involved with higher administration. However, he said: "they backed me on all those decisions, and they took the blows if there was a backlash." During his very first month in the position, he had to eliminate a 
program. Though apprehensive about reactions to this decision, he was able to accomplish it by collaborating with provosts, president and chancellor, and by frequently updating and explaining circumstances to the board of curators. He shared about this experience, "It gave me a lot of faith that I can do this. It was incredible." Comparable to Theodore's anecdote, Anthony recounted trying to ensure all potentially relevant leaders are in agreement with his management of challenging situations. Marco created strong relationships with his dean, associate dean, and other chairs within his college. For this, he believes he has received robust support from them, and they, in turn, provide all the resources he asks for.

Yet, there is an abundance of unknowns in the diverse set of areas when a chair begins the job. The strategy that chairs have picked up was to respectively consult diverse set of professionals like financial officers, human resources staff, their administrative assistant, and associate deans. New chairs approach them often with the questions they run into. Unlike leadership alliances in which chairs approach leaders to gain their approval on planning decisions and securing their support and alliance, in consultation, the chairs would approach anyone to collect data, learn about an issue and ask their questions on a matter. Almost all chairs use this strategy when faced with a new challenge or ambiguous situation. Anthony seeks assurance from multiple sources when he encounters a problem. He first approaches his associate dean for consultation, and then if he is unsatisfied or in question with the answer he receives, he will seek his dean's advice also. He holds the guidance given by his chair's advisory board in high esteem, as it is made up of many disciplines with a vast array of accumulated knowledge. Genevieve seeks guidance from many people to answer her questions, including the fiscal officer, human resources, and provost's office staff. She is confident in the success of her approach and believes she has been supported by those she has reached out to. Barbara and 
Marco likewise mentioned that they often work closely with many people (e.g., associate deans, senior faculty, fiscal and HR officers) to explore solutions to their difficulties.

\section{Summary}

This chapter delineated the lived experience of the new department chairs using the four tenets of transition theory of Schlossberg $(1981,1995,2011)$, self, situation, support, and strategy. I explored the self facet of the chairs in transition by disclosing their roles, responsibilities, and aspirations. I then delved into the situation of the transition by focusing on the department culture and relationship of the new chairs, official transition procedures, and the challenges of this transition. Then, the focus was on the support systems that were provided to the chairs and what strategies chair utilized to navigate their transition.

Essentially the findings in this chapter revealed that chairs walked into the role with different type of aspirations and goals comparing faculty and the success metrics for chairs are widely distinct from their previous role. Each chair in this study is undergoing a unique professional and psychological journey, and they are making sense of this event different from a personal and professional standpoint. Chairs expressed that they felt they felt blindsided and they underestimated the learning curve of some vital responsibilities like human resource issues or academic finance and advancement outreach. In the transition, predecessors of chairs influenced this journey greatly. In order to navigate this leadership role's challenges, the new chairs learned that they should ally with their leaders and consult vulnerably with field experts. Finally, chairs unanimously stated that the single most important quality for this role is interpersonal skills and the ability to manage different types of people. 
Building upon these findings, in the next chapter I will answer the research questions by interpreting the findings, and I will elaborate the implications of the study. I will also provide the limitations and propose some ideas for further research in this area. 


\section{CHAPTER 5: DISCUSSION AND IMPLICATIONS}

The purpose of this case study is to explore the transition of faculty members to chair role at the University of Missouri. Most tenured faculty members at American higher education institutions at some point in their career take an academic leadership role (Finkelstein, Conley \& Schuster, 2016), thus it is important to understand how this transition takes place to facilitate organizational success. The career transition to leadership is one of the most formidable challenges at the individual and organizational level (Howard, 2003).

I approached this study from a qualitative approach to gain in-depth understanding of the entire experience of the transition, including its complexities and challenges. The case study design was used to provide an intensive bounded analysis of the real-life transition experience of chairs at University of Missouri. Case study design empowered the methodology by encompassing departmental and institutional context while taking into account each participant's idiosyncratic differences. This study utilized constructivism epistemology to capture the meaning-making of the various realities that new chairs experienced through interacting with their social worlds (Merriam, 1998). The data were collected from the seven chairs through various data sources, including two rounds of interviews, 2-week calendars, leadership appointment letters, and resumés, in order to have representative data that could be triangulated across these sources.

Throughout this study, I sought to answer the following research questions:

1. How do new department chairs experience transition from a faculty position to a leadership role?

2. How do new department chairs navigate the challenges of the transitioning to leadership? 
In this chapter, I will answer these research questions based on the findings, existing literature, and the conceptual framework. I will suggest the implications of this study on current policy and practice of departmental leadership at the institutional level and beyond. Finally, I elaborate on the limitations of this research, and provide suggestions for the future research in this area.

I utilized Schlossberg's transition theory $(1981,2011)$ as the guiding conceptual frameworks to discuss how the findings of this case study help answer stated these research questions. Answers to these research questions were enlightened by the principles of constructivism and will provide a pathway to discuss implications of this study.

\section{Transition Experience to Leadership}

In response to the first research question, this section focuses on the transition experience. Transition to leadership for faculty is a metamorphosis, as explained in Theme I: Transition is a Multifaceted Departure. This experience is a radical transformation of career aspiration and socio-organizational identity. Prior to committing to this experience, these individuals who had been pursuing a teaching and research career, pivoted toward higher level aspirations to more directly impact the greater educational space. The findings of this research support Palm (2006) result that chairs who accept the position are willing to sacrifice their academic career to further the goal of department and college.

In some cases, participants accepted this role because they were nominated by their peers, and knowing the challenges, they selflessly stepped out of their comfort zone to serve. The transformation is felt greatest in their day-to-day activities. Theme I: Transition is a Multifaceted Departure explained the loss of autonomy as a scholar, who, now bounded to the office, frequents meetings and holds the regular workhours of a manager. Chairs move away from their 
previous identity as a professor who generates knowledge, to someone who must practice the art of persuasion, management, and compromise (Gmelch \& Parkay 1999). Chairs function as managers when they are working on processes, structures, and paperwork; and as leaders while working on culture, mission, and people (Bowman, 2002).

Theme II: Leadership is Interpersonal, demonstrated how new chairs' professional social circle and power dynamics were altered. Chairs attempt to socio-organizationally adopt to this new managerial role (Gmelch, 2004). In this role, they began interacting with different constituencies and felt that they were perceived differently. One of the interviewed chairs stated that she is now "the voice of administration" or as Palm (2006) phrased it, the chair moves to the dark side from the faculty's point of view. Throughout the process, the chair understands the value and behavior expectations that they must acquire, and the disposition needed to perform the role (Smith \& Stewards, 1999). During this early transition and adaptation stage, chairs become conflicted. They experienced role ambiguity within their value system as faculty, as well as their administrative responsibilities, which were always not well-articulated. (Gmelch \& Parkay 1999).

For faculty members, success metrics were clearly defined in terms of research productivity and teaching performance, as discussed in the Subtheme: Altered Success Metrics. In the early stages of leadership, new chairs felt successful in their role when making department faculty and supervisors satisfied. Carrol and Wolverton (2004) found that while chairs are confused about how they are being evaluated by their supervisors, Smith and Steward (1999) report that faculty members scrutinized and criticized every action of new chairs. Lord and Hall (2005) reported that novice leaders are sensitive to social feedback and reactions. Validation by 
others plays an important role in their confidence building, since they want to feel accepted by others and to be viewed as a leader.

Theme III: Chairs are Finding their Way, showed that among the various environmental and situational factors, predecessors have the greatest impact on the new chair's transition experience. This theme also directly reflects the Schlossberg $(1981,2011)$ 's coping factors of strategies and support. Incumbent chairs have immense latitude in supporting the conditions of transition by involving the incoming chair during decision making processes regarding important departmental issues and orientating and familiarizing new chairs with role responsibilities. New chairs who shadowed their predecessors and received mentorship were found to be more confident in first few months of their new role and felt less stress before the start date than their peers. Shadowing opportunities also played an important role in the organizational socialization of chairs during the early stages of the transition, assisting the smooth assimilation and socialization with the anticipated group of new constituencies and participants among their old peer group (Smith \& Steward, 1999). In a protected environment, they could gradually learn the expected values and behaviors. More or less, new chairs perceived their workspace collegial, healthy, and supportive, noting a main contributor to this being the democratic election and nomination process of chairs in the institution.

While participant chairs in this study all began their careers as tenured faculty and their transitions had many commonalities, their election processes varied widely, as discussed in the Subtheme: Multiple Paths, One Destination. They experienced varying levels of support from predecessors, and the appointment timeline and its uncertainties, looked different as their assessment of the event varied. This finding is in line with Nicholson (1984). Nicholson suggested that idiosyncrasies are important aspects of transition, as different people adjust to 
changing lifestyles, role expectations and skill requirement with different outlooks. How a candidate perceives themselves and the event shapes their journey, sensemaking processes, and coping mechanisms. Some chairs in the study perceived the transition as a temporary experience, and others viewed it as a permanent departure from their role as a professor. Some perceived it as a great opportunity to hone their skillset, while others considered this role a threat to their scholarships. Schlossberg (1981) stated that how the transition fits within an individual's stage, situation, and style at the time of the transition, shapes the transition but not the transition event itself.

\section{Navigation of Challenges}

In response to the second research question, this section will cover how chairs navigated the challenges of transition. The pillars of support and strategies of transition model (Schlossberg 1981, 2011), focuses on coping mechanisms and the navigation of challenge. As explained in Theme I: Transition is a Multifaceted Departure, during the early stages of their transition, new chairs felt overwhelmed, uncertain, and occasionally unprepared. During the early days, chairs learned the jurisdiction and range of duties, and reported feeling uncomfortable, especially as their scholarly credibility and core competencies of research and teaching do not directly translate to the requirements of this role. Aziz et al (2005) identified the categories required for a chair to perform, including budgeting and resource allocation, curriculum and programs, department administration, external communication, faculty affairs, internal communication, legal, office management, professional development, staffing, and student affairs. This study supports the findings of Aziz et al. In Subtheme: Trial by Fire, participants of this study shared that they felt blindsided by the complexity of the role and their lack of preparation in many areas, including higher education finance, human resources, and advancement. Some individuals 
stressed shortcomings like legal matters, institutional policies, staffing and rhetoric. In general, new chairs at the University of Missouri received minimal training, if any, and did not have exposure to structured development programs before assuming the role except few cases of shadowing the predecessor.

While Mahlanhu (2015) found that the most important competency deficiency is interpersonal skills in supervisory roles, it was not the case among the participant chairs. As shown in Theme II: Leadership is Interpersonal, new chairs capitalized on their interpersonal skills to navigate the challenges they faced in other areas. Faculty members have the expectation that their chairs operate more as a support rather than as a leader (Bowman, 2002). Chairs reported that it is unlikely that they would have been elected by their colleagues, had they not been in good standing with them. Griffith (2006) stated that the new relationship dynamics with old coequal colleagues, puts chairs in a unique position to make decisions that affect their colleagues, and at times can create some friction. With that, the quality and nature of their relationships can undergo scrutiny by all constituencies. This study found the similar changes in dynamics after appointment.

Theme III: Chairs are Finding their Way, showed that the service portion of faculty work distribution provided an initial window into academic leadership. As faculty, they were involved in different taskforces and councils, both on and off campus, where they had exposure to academic administration and interdepartmental connections. In this study similar to Ibarra (1999), I found that novice leaders adapt and change through the social feedback of others. They solidify their self-view and modify their strategy based on their reactions of their influential figures in their workspace; especially when those figures are in the position to evaluate the performance. Granted when there was sufficient time and willingness, predecessors offered 
hands-on coaching opportunities, mentorship, shadowing, and exposure to departmental leadership to the incoming chair. In the anticipated transition phase and before official start date, predecessors provide practical skills and knowledge orientation and provide socio-organizational assimilation.

Lord and Hall (2005) stated that leaders develop leadership skills by observing the behavior of other leaders. They learn the normative behavior of how a leader should act, in order to be perceived as a leader by others. The Theme III: Chairs are Finding their Way, showed that after the official start date, chairs attended regular meetings with their deans, associate deans, other leadership personnel. These meetings provided chairs networking, organizational socialization, and exposure to the leaders who carry years of institutional knowledge and practical expertise. In that line, Hernz-Broome \& Hughes (2004) found that leaders have a major trend of learning through coaching, mentorship and action learning, rather than formal training courses or seminars.

Problems and challenges are inevitable in a leadership role; even more so for new leaders (Schlossberg, 2011). New chairs use their alliances with leadership and consultation with experts, as their two main strategies to overcome challenges. To navigate the challenges and turmoil of a department chair's job, participants were quick to learn that they needed to create allyship with the senior leadership, to garner support during difficult decisions. To do so, chairs would share their plans with their leaders, and after securing approval, they would move to execute the agreed upon plans and decisions.

\section{Implications for Policy and Practice}

This study informs administrators, policy makers, and other individuals involved in leadership development across higher education institutions about the transition process into 
leadership. The findings of this study have multiple policy and practice implications for chairs, academic leaders and policymakers at the University of Missouri, and beyond. Considering the wide range of duties assigned to a department chair, Aziz et al. (2005) and Bryman (2007) have expressed that many higher education institutions should be more informed about the challenges facing new chairs, and the support needed to be successful.

Building on the principle of the human capital theory and the necessary competencies of a chair (Aziz et al. 2005), the first step in designing a support system is undersanding the process. Thus, it demands assessment of these needs and challenges (Noe, 2013). Awareness of the needs and challenges is in line with the needs' assessment approach that Noe (2013) recommended when designing a competency development program. Themes III: Chairs are Finding their Way, revealed competency gaps in how chairs currently learn through formal courses, seminars, workshops, and shadowing. As discussed in this study, and previously shown by Foster (2006), leading a department for the first time is a unique experience. The transition from being a professor and researcher, to a leader and manager, demands a distinct skillset. While each experience is unique, the findings of this study will inform chairs about what to expect, the common challenges that they might face, and the strategies previous chairs used to navigate them. This knowledge helps prepare them and gives them strategies to utilize to seek out relevant support.

Gmelch (2004) discussed the cost of transition to the institution and chairs. University leaders and policy makers can design a transition experience that minimizes the stress and tension for chairs and reduces cost for the university. The support from predecessors that new chairs received before beginning their role was described in Theme III: Chairs are Finding their Way. Predecessors can develop a plan for shadowing, mentorship, and onboarding. One 
takeaway of this study is the significance of the predecessors-incumbent chair relationship. The Theme III: Chairs are Finding their Way explained that an existing chair carries huge amount of institutional knowledge necessary for the coming chair to be aware of. Also, predecessors have gradually learned the necessary skills needed by the next chair through shadowing, mentoring, and coaching. The predecessor plays a crucial role in the support system for new chairs, both before and after the official handover of responsibilities. In a few cases, predecessors could prepare their successors when they speculated, who would be appointed. With that belief, these predecessors initiated a succession plan by providing an abundance of exposure through leadership meetings, collaboration on important decision, and frequent coaching sessions.

The timing of the nomination, election, and official appointment need to be revaluated by policymakers. Theme I: Transition is a Multifaceted Departure showed that all the chairs in this study were appointed to the role a semester before the start date. Earlier appointments provided more time for the prospective and incumbent chairs to meet, shadow, and become acclimated. This policy must consider the formal duty distribution of incumbent chairs, so prospective chairs can adjust in a way that allows then to invest in the transition. The gradual hand-over of responsibility will end in the final semester. Based on the workplace socialization of Smith and Steward (1999), the three stages are anticipation, encounter, and assimilation. During the anticipation stage, the chairs have yet to have been formally appointed to the position. The chairs can shadow and take advantage of the various training courses and workshops on administration issues. Through activity exposure, they also learn by attending leadership meetings and participating in administrative and managerial tasks of the department.

Idealistically, the University of Missouri can develop am succession plan for the incoming chairs. Currently each department creates their own way transition procedure. After 
appointment some deans and associate deans meet and provide leadership, but it is currently the sole responsibility of the chairs to prepare themselves. Leaders and policymakers at higher levels of the university must make informed decisions about how to design an effective support system for their new chairs. Awareness of the transition journey and challenges on this particular campus is the first step in that direction. This study portrays the journey and current navigational strategies of the individuals who embark on this path and should draw the attention of decisionmakers.

As explained in Theme II: Leadership is Interpersonal, chairs participating in this study declared this role as isolating one. Some of them had difficulties finding a community of chairs who can support each other. This study will show them that they are not the only ones grappling with the challenges that they face. Knowing that others are facing some of the same issues during the transition, especially in the early stages, can be reassuring. It can boost their confidence in their own potential and shield them against the negativity and feelings of incompetency. In addition to being aware of the potential challenges, chairs will also learn from the strategies other chairs have utilized to navigate them.

I have shown the complexity of this role and transition. This research informs and encourages prospective chairs to look beyond the formal job description, tasks, and duties. Throughout higher education, administrators should recognize the complexity of the chair role and the typical route does not provide training. There is a need for more systematic support to elevate their efficiency during the first years of the job and increase the quality of both professional and personal life. The pressure and stress of this job spills over to their personal lives and can impact their work performance, as well as life quality. 


\section{Implications for Further Research}

The findings of this research expand the extant literature on academic leadership, higher education administration, and leadership development. I sought to address multiple research gaps with this study. There have been calls by other scholars for further research in this area. I address the call of Hetch (2001), Gmelch (2004), and Aziz et. al (2005) that there is a need for more research on academic leadership and development. There are several implications for research from this study and multiple future studies that could be conducted to further understand transition into academic leadership.

The majority of the research on the chairs is the result of the ending periods reflection and retrospective thoughts by chairs on their experience. It expands beyond autoethnographic studies such as of Palm (2006). No previous literature has yet to capture the experience of chairs in the early stages of their transition into the role. Gillet-Karam (1999), Hubbell and Homer (1997), Seagren (1993), Thomas and Schuh (2004) and Palm (2006) addressed role challenges, competency, and skill deficiencies, but they did not capture transition in the early stages. This

study expanded the scope of transition to leadership with Theme I: Transition is a Multifaceted Departure, to prior to the official appointment of the department chair. Through a constructivist approach, I tapped into the various stage of sense making in transition in Theme I: Transition is a Multifaceted Departure.

The peculiarity of this case study provides this opportunity for other researchers to study the transferability of the findings in similar institutions, and to compare the findings in different type of universities. Replication in similar types of institutes will address the credibility and dependability of the findings to other public research universities. Finally, replication in other 
types of universities will determine how specific are the findings and what findings stand true regardless the type of institution.

This study discovered that predecessors shape the transition and are irreplaceable resources for chairs in navigating both the predictable and unpredictable challenges. Due to its importance, future research should tease out the effect of predecessors and explore effective succession plans, highlighting their key success factors and challenges, and provide a comprehensive succession plan for the coming chairs.

Transformation of chairs' social circle and their interaction patterns with different constituencies should be explored. This social network analysis of chairs can bring value to this space by empirically studying the connections, distribution, and segmentation of their interactions through quantitative studies analyzing the amount of phone and email and how it changes among different types of constituencies. Longitudinal case studies to investigate the chairs' evolution of thoughts, feelings, and various skills in the course leadership will also be valuable to capture their professional journey.

Schlossberg's transition theory $(1981,1995,2011)$ was a valuable framework. However, the categories of self and situation demonstrate how support and strategy are interdependent. This, future researchers need to be aware of, and consider the effect of each category on the others. For instance, self of a person will influence the situation, and vice versa. Sometimes the line between the two becomes blurry. Considering these variables on a continuum of locus of control can be an improvement on this model. For example, some factors are more inclined toward self-side of locus of control, and others to the situation-side which is external locus of control. 


\section{Conclusion}

This is a unique case study that focuses on a particular subset of American higher education institutions. This research is an effort to fill the gap of knowledge in the general literature. To date, this is the first study to use Schlossberg's transition theory $(1981,1995,2011)$ to explore the transition of faculty members to leadership, and systematically study four core pillars of the transition: self, situation, support and strategies. The findings of the study revealed multiple sense making processes of chairs in transition and the subjective understanding of transition.

Hetcht (2001) asserted that chairs oftentimes find themselves "operating in a murky landscape of either ill-defined or conflicting expectations" (p. 7). A leadership role is a stressful job that deals with interpersonal conflict, bureaucracy, and adverse impact on their research (Carroll \& Wolverton, 2004), causing chairs to feel greater degree of stress and loss of identity compared to faculty (Gmelch, 1991). While this research found those as challenges, this study revisited and expanded on some common and idiosyncratic challenges and described how chairs navigated them.

Department chairs hold roles of authority, decision making and the power to shape the policies and practices within their departments and colleges. In the larger scheme of higher education, chairs and faculty life directly impact students' success, retention, institutional sustainability and indirectly impacts community and societal goods. This study explored transitioning into academic leadership and navigating its challenges to improve this aspect of higher education administration and leadership. 


\section{REFERENCES}

Alstete, J. W. (2000). Posttenure faculty development: Building a system for faculty improvement and appreciation. ASHE-ERIC Higher Education Report, 27(4), 22-56.

Arthur Jr, W., Bennett Jr, W., Edens, P. S., \& Bell, S. T. (2003). Effectiveness of training in organizations: A meta-analysis of design and evaluation features. Journal of Applied psychology, 88(2), 234-245.

Aziz, S., Mullins, M. E., Balzer, W. K., Grauer, E., Burnfield, J. L., Lodato, M. A., \& CohenPowless, M. A. (2005). Understanding the training needs of department chairs. Studies in Higher Education, 30(5), 571-593.

Badawy, M. K. (1995). Developing managerial skills in engineers and scientists: Succeeding as a technical manager: John Wiley \& Sons.

Baker, L., Leslie, K., Panisko, D., Walsh, A., Wong, A., Stubbs, B., \& Mylopoulos, M. (2018). Exploring faculty developers' experiences to inform our understanding of competence in faculty development. Academic Medicine, 93(2), 265-273.

Bates, R. (2004). A critical analysis of evaluation practice: The Kirkpatrick model and the principle of beneficence. Evaluation and Program Planning, 27(3), 341-347.

Baxter, P., \& Jack, S. (2008). Qualitative case study methodology: Study design and implementation for novice researchers. The Qualitative Report, 13(4), 544-559.

Berdrow, I. (2010). King among kings: Understanding the role and responsibilities of the department chair in higher education. Educational Management Administration \& Leadership, 38(4), 499-514.

Biddle, J., \& Roberts, K. (1994). Private sector scientists and engineers and the transition to management. Journal of Human Resources, 29(1), 82-107. 
Bland, C. J., \& Risbey, K. R. (2006). Faculty development programs. Effective Practices for Academic Leaders, 1(7), 1-16.

Bowman Jr, R. F. (2002). The real work of department chair. The Clearing House, 75(3), 158162.

Bowen, G. A. (2009). Document analysis as a qualitative research method. Qualitative research journal, 9(2), 27-40.

Bridges, W. (2004). Transitions: Making sense of life's changes. Da Capo Lifelong Books.

Bryman, A. (2007). Effective leadership in higher education: A literature review. Studies in Higher Education, 32(6), 693-710.

Burke, L. A., \& Hutchins, H. M. (2008). A study of best practices in training transfer and proposed model of transfer. Human Resource Development Quarterly, 19(2), 107-128.

Carroll, J. B., \& Wolverton, M. (2004). Who becomes a chair? New Directions for Higher Education, 2004(126), 3-10.

Centra, J. A. (1978). Types of faculty development programs. The Journal of Higher Education, $49(2), 151-162$

Creswell, J. W. (2013). Research design: Qualitative, quantitative, and mixed methods approaches ( $4^{\text {th }}$ ed.). Sage Publications.

Denzin, N. K., \& Lincoln, Y. S. (2002). The qualitative inquiry reader. Sage Publications.

Draganidis, F., \& Mentzas, G. (2006). Competency based management: A review of systems and approaches. Information Management \& Computer Security, 14(1), 51-64.

Eble, K. E., \& McKeachie, W. J. (1985). Improving undergraduate education through faculty development. An analysis of effective programs and practices. ERIC.

Finkelstein, M. J., Conley, V. M., \& Schuster, J. H. (2016). The faculty factor: Reassessing the 
American academy in a turbulent era. JHU Press.

Foster, B. L. (2006). From faculty to administrator: Like going to a new planet. New Directions for Higher Education, 2006(134), 49-57.

Gappa, J. M., \& Austin, A. E. (2010). Rethinking academic traditions for twenty-first-century faculty. AAUP Journal of Academic Freedom, 1(1), 1-20.

Gillet-Karam, R. (1999). Preparing department chairs for their leadership roles. New Directions for Community Colleges. 105, 1-3.

Glick, M. D. (2006). Becoming "one of them" or "moving to the dark side". New Directions for Higher Education, 2006(134), 87-96.

Gmelch, W. H. (1991). Paying the Price for Academic Leadership: Department Chair Tradeoffs. Educational Record, 72(3), 45-48

Gmelch, W. H., \& Parkay, F. W. (1999, April). Becoming a department chair: Negotiating the transition from scholar to administrator. Paper presented at the Annual Meeting of the American Educational Research Association, Montreal, Ontario. Retrieved August 14, 2006, from

http://www.eric.ed.gov/ERICDocs/data/ericdocs2/content storage 01/0000000b/80/11/7 e/62.pdf

Gmelch, W. H. (2004). The department chair's balancing acts. New Directions for Higher Education, 2004(126), 69-84.

Griffith, J. C. (2006). Transition from faculty to administrator and transition back to the faculty. New Directions for Higher Education, 2006(134), 67-77.

Hecht, I. W. (2001). 2: Transitions and transformations: The making of department chairs. To Improve the Academy, 19(1), 17-31. 
Hernez-Broome, G., \& Hughes, R. J. (2004). Leadership development: Past, present, and future. Human Resource Planning, 27(1), 24-32.

Howard, C. A. (2003). From engineer to manager: A qualitative study of experiences, challenges, and individual transitions for engineering managers in aerospace companies (Doctoral dissertation). Available from ProQuest Dissertations \& Theses Global database.

Hubbell, L., \& Homer, F. (1997). The academic department chair: The logic of appeasement. Political Science \& Politics, 30(2), 209-213.

Ibarra, H. (1999). Provisional selves: Experimenting with image and identity in professional adaptation. Administrative Science Quarterly, 44(4), 764-791.

Johnson, D., \& Sargeant, A. (1998). Motives for transition: An exploratory study of engineering managers. Human Resource Management Journal, 8(3), 41-53.

Kirkpatrick, D. L. (1996). Invited reaction: Reaction to Holton article. Human resource Development Quarterly, 7(1), 23-25.

Lazarus, R. S., DeLongis, A., Folkman, S., \& Gruen, R. (1985). Stress and adaptational outcomes: The problem of confounded measures. American Psychologist, 40, 245-254

Lincoln, Y. S., \& Guba, E. G. (1985). Naturalistic inquiry (Vol. 75). Sage.

Lord, R. G., \& Hall, R. J. (2005). Identity, deep structure and the development of leadership skill. The Leadership Quarterly, 16(4), 591-615.

Mahlangu, S. R. K., \& Govender, C. (2015). Management competencies required in the transition from a technician to a supervisor. SA Journal of Human Resource Management, 13(1), 1-8.

Martin, H. J. (2010). Workplace climate and peer support as determinants of training transfer. 
Human resource development quarterly, 21(1), 87-104.

Merriam, S. B. (1988). Case study research in education: A qualitative approach. Jossey-Bass.

Merriam, S. B. (2009). Qualitative Research: A Guide to Design and Implementation. John Wiley \& Sons.

Morrow, S. L. (2005). Quality and trustworthiness in qualitative research in counseling psychology. Journal of counseling psychology, 52(2), 250-260.

Nicholson, N. (1984). A theory of work role transitions. Administrative science quarterly, 29, 172-191.

Noe, R. (2012). Employee Training \& Development (6 ${ }^{\text {th }}$ ed.), McGraw-Hill.

Palm, R. (2006). Perspectives from the dark side: The career transition from faculty to administrator. New Directions for Higher Education, 2006(134), 59-65.

Patton, M. Q. (2014). Qualitative Research \& Evaluation Methods: Integrating Theory and Practice, Sage Publications.

Potgieter, I., Coetzee, M., \& Basson, J. (2011). Management competencies for the development of heads of department in the higher education context: A literature overview. South African Journal of Labour Relations, 35(1), 81-103.

Praslova, L. (2010). Adaptation of Kirkpatrick's four level model of training criteria to assessment of learning outcomes and program evaluation in higher education. Educational Assessment, Evaluation and Accountability, 22(3), 215-225.

Roberts, K. (1994). The transition into management by scientists and engineers: a misallocation or efficient use of human resources? Human Resource Management, 33(4), 561-579.

Salas, E., Tannenbaum, S. I., Kraiger, K., \& Smith-Jentsch, K. A. (2012). The science of training and development in organizations: What matters in practice. Psychological Science in the 
Public Interest, 13(2), 74-101.

Savin-Baden, M., \& Major, C. H. (2012). Qualitative Research: The Essential Guide to Theory and Practice. Routledge.

Schlossberg, N. K. (1981). A model for analyzing human adaptation to transition. The counseling psychologist, 9(2), 2-18.

Schlossberg, N. K. (1995). Counseling adults in transition: Linking practice with theory. Springer Publishing Company.

Schlossberg, N. K. (2011). The challenge of change: The transition model and its applications. Journal of Employment Counseling, 48(4), 159-162.

Seagren, A. T. (1993). The Department Chair: New Roles, Responsibilities and Challenges. ASHE-ERIC Higher Education Report No. 1: ERIC.

Seedorf, R. G., \& Gmelch, W. H. (1989). The Department Chair: A Descriptive Study. Paper presented at the American Educational Research Convention, San Francisco.

Seidman, I. (2013). Interviewing as qualitative research: A guide for researchers in education and the social sciences: Teachers College Press.

Smith, A. B., \& Stewart, G. A. (1999). A statewide survey of new department chairs: Their experiences and needs in learning their roles. New Directions for Community Colleges, 1999(105), 29-36.

Stake, R. E. (1995). The art of case study research. Sage Publications.

Stake, R. E. (2013). Multiple case study analysis. Guilford Press.

Steinert, Y. (2000). Faculty development in the new millennium: Key challenges and future directions. Medical Teacher, 22(1), 44-50.

Thomas, J. R., \& Schuh, J. H. (2004). Socializing new chairs. New Directions for Higher 
Education, 2004(126), 11-25.

Tucker, A. (1984). Chairing the academic department: leadership among peers (2nd ed.). ACE/Macmillan.

Ward, K. (2003). Faculty service roles and the scholarship of engagement. Eric Digest.

Wentland, D. (2003). The strategic training of employee's model: Balancing organizational constraints and training content. SAM Advanced Management Journal, 68(1), 56-72.

Willis, C. L. (2010). To the dark side and back: The administrative odyssey of an academic sociologist with lessons learned. The American Sociologist, 41(2), 190-209.

Wolverton, M., Ackerman, R., \& Holt, S. (2005). Preparing for leadership: What academic department chairs need to know. Journal of Higher Education Policy and Management, $27(2), 227-238$.

Wolverton, M., Gmelch, W. H., Wolverton, M. L., \& Sarros, J. C. (1999). A comparison of department chair tasks in Australia and the United States. Higher Education, 38(3), 333350.

Yin, R. K. (2013). Case study research: Design and methods. Sage Publications. 


\section{APPENDIX I: INVITATION EMAIL}

Dear Dr.

I am a PhD candidate in Educational Leadership and Policy Analysis (ELPA) at the University of Missouri. I have heard many amazing news about you of my friends and colleagues, and recommended you as an ideal individual who can invaluably contribute to my dissertation research.

My research, co-advised by Dr. Jeni Hart and Dr. Bradley Curs, explores the transition experience of early-career chairs from professoriate into leadership roles. My dissertation aims to understand how faculty members of the University of Missouri experience, perceive and make sense of their transition into leadership, as well as their ability to navigate the challenges of the new position.

If you are interested in participating, you will be invited to two interviews (approximately 60 minutes each). Prior to the interviews:

- We will schedule the first interview (may be conducted in-person, via Zoom, telephone, etc.) based on your availability.

- I will e-mail you informed consent and confidentiality agreements that explain the full details of participation in this study.

- To better understand the nature of your role as a chair, I would like to collect an anonymized typical weekly calendar of your as a chair.

I appreciate your consideration of my request. Please feel free to contact me with any questions you may have. Should you be interested in participating in the study, our next step is to schedule an interview. I look forward to hearing from you.

Kind regards, Matin

\section{Matin Qazi}

Doctoral Candidate | Department of Educational Leadership \& Policy Analysis Master's Student | School of Information Science \& Learning Technologies

Graduate Assistant | Faculty Institute for Inclusive Teaching University of Missouri

202 Hill Hall, Columbia MO, 65211

Phone: (573) 808-7971

$\mathrm{He} / \mathrm{Him}$ pronouns 


\section{APPENDIX II: INFORMED CONSENT}

You are invited to join a research study titled "transition experience of professors into academic leadership positions". The participants must be first-time department chairs at the University of Missouri who are in their first or second year in position. I am a doctoral candidate in the Educational Leadership \& Policy Analysis department at the University of Missouri. In this qualitative research study, I am investigating the experiences of a new department chairs to unveil how faculty experience, perceive, and make sense of their process of transition into department chair. In this case study, I will essentially try to answer how new department chairs experience challenges in transition from a faculty position to an administrative role, and how new department chairs navigate challenges of the transition.

\section{Prior to the first interview the researcher will collect the following documents:}

- Your current resume or curriculum vitae to assist in preparation for the interviews.

- Your calendar of two typical weeks without details and individuals' name.

- The job positing for your position or your letter of appointment that I can see your official job description and responsibilities.

- The performance evaluation rubrics of your position and department policy manual.

RISKS: This study involves no physical risks. This study has the potential to involve psychological risks should emotions arise as participants share their story. There may also be other risks that we cannot predict. You can stop participating at any time.

CONFIDENTIALITY: I will take the following steps to keep information about you confidential, and to protect it from unauthorized disclosure, tampering, or damage:

- You will be assigned a pseudonym to protect your privacy. I will ensure confidentiality to the best of my ability in the final version of the dissertation and future publications.

- Steps will be taken to protect electronic data (pre-interview questionnaire, CV, audio files of interviews and other documents). Files will be immediately transferred to a password protected computer on a secure network. The master list of participant names and assigned pseudonyms will be stored in a locked file cabinet. 
- Only the principal investigator will have access to the above listed information. Should I have questions about my data analysis, I would only share hard copy interview transcripts with my doctoral supervisor. Original, electronic files will never be shared.

- You will have an opportunity to review the interview transcripts and identify any components that you think would be personally identifying. For my dissertation and future publications, I will do my best to use quotes that are not personally identifying, or I will alter quotes as needed to remove any specific names or institutions.

YOUR RIGHTS AS A RESEARCH PARTICIPANT? Participation in this study is voluntary. You have the right not to participate at all or to leave the study at any time. Deciding not to participate or choosing to leave the study will not result in any penalty or loss of benefits to which you are entitled, and it will not harm your relationship with the principal investigator. Should you have questions or concerns about the study, you may contact Matin Qazi, Principal Investigator, at (573) 808-7971 or mqk95.@mail.missouri.edu. You may also contact my doctoral advisor, Dr. Bradley Curs at (573) 882-2759 or cursb@missouri.edu. Should you have questions regarding your rights as a human subject research participant, you may contact the Institutional Review Board (IRB) at the University of Missouri at 573-882-3181 or irb@missouri.edu.

\section{By signing my name below, I confirm the following:}

- I have read/had read to me this entire consent form.

- All of my questions were answered to my satisfaction.

- The study's purpose, procedures/activities, potential risks and possible benefits were explained to me.

- I voluntarily agree to take part in this research study. I have been told that I can stop at any time. 


\section{APPENDIX III: INTERVIEW PROTOCOL: SESSION I}

- What prompted you to become a faculty member?

- Please tell me about your professional life when you were faculty? [How is your experience in being a faculty?]

- Tell me about what performance evaluation look like for a faculty?

- Tell me about the culture of your department?

- How often you meet as a department? Tell me about your department meetings?

- What was your responsibilities as a faculty?

- What prompted you to become a department chair? [And how that happened? What was your motivation to be a chair?]

- How was the procedure of selection and appointment? [Did you have to compete with other people?]

- How do you describe your experience being a department chair and in a leadership role?

- Please tell me about the transition period. [Was it anticipated or Unanticipated event]

- How was your perception when it happened? How did you feel about it then?

- What are the differences in being a chair comparing to a faculty?

- How your relationships have changed with colleagues and other people since you become a chair?

- How your day to day at work has changed since you become a department chair?

- What did blindsight you? [What did you not expect since you have been a chair?

- What are you challenges in general?

- Was there a demarcation when you become the chair?

- What do you like about this role?

- What do you dislike about this role?

- Who do you talk to as part of your job as a chair?

- Can you give me a story that you made the most difference or contribution? 


\section{APPENDIX IV: INTERVIEW PROTOCO- SESSION II}

- Explore issues discussed in the previous session that need expansion or clarification

- Please walk me through your transition?

- How did you navigate from being a faculty into administration world? What is it like for you?

- How are you dealing with the challenges of department chairs? [How are resolving these issues?]

- How and from whom or what resources do you get help?

- Can you walk me through your problem-solving behavior?

- How are learning to solve problems that you encounter as a chair?

- Can you tell me about interactions as a chair? How do you manage with personal emotional issues?

- What are the top three challenges that you have experienced as a department chair? How are you dealing with them?

- What prepared you for the transition? [What were the learning opportunities before officially starting the position?]

- How did you prepare for the department chair position?

- How are you processing it and making sense of them? What skills do think department chairs ideally should have?

- Which of these skills do you feel proficient in? How have you learned these skills that you feel you have masters? Before you being a department chair, and while you were the department chair?

- What kind of formal and informal training did you receive?

- What skills do you think you can develop more?

- What advice you have for new department chairs?

- Any other topic that you think I need to know? 


\section{VITA}

Matin Qazi was born in 1984 in Gonbadekavoos, Iran. He received his Bachelor of Arts degree from Arak University in English Literature. Afterward, he simultaneously pursued Master of Arts in Teaching English as Foreign Language (TEFL) at Arak University and his Master of Business Administration at Payame-Noor University. Upon completion of his compulsory military service, he followed his dream to earn a doctoral degree in the United States. While doing his $\mathrm{PhD}$ in Educational leadership and Policy Analysis, he completed his studies for Master of Science in Learning Technologies and Design. Currently, he is a User Experience researcher at a Fortune 500 company. 